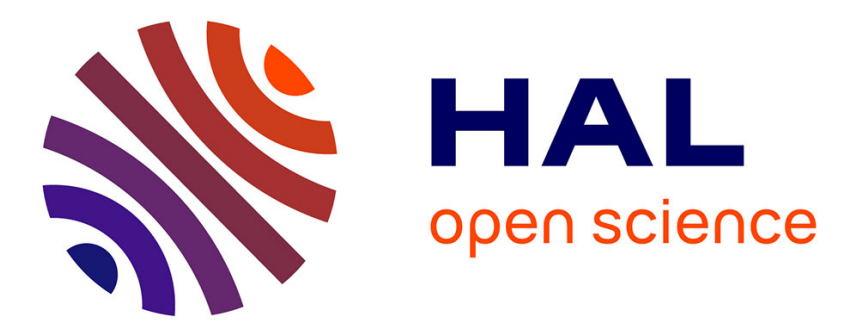

\title{
Modelling of the microstructure of mesoporous alumina constrained by morphological simulation of nitrogen porosimetry
}

Haisheng Wang, Dominique Jeulin, François Willot, Loïc Sorbier, Maxime Moreaud

\section{To cite this version:}

Haisheng Wang, Dominique Jeulin, François Willot, Loïc Sorbier, Maxime Moreaud. Modelling of the microstructure of mesoporous alumina constrained by morphological simulation of nitrogen porosimetry. Colloids and Surfaces A: Physicochemical and Engineering Aspects, 2018, 553, pp.378 - 396. 10.1016/j.colsurfa.2018.05.043 . hal-01821865

\section{HAL Id: hal-01821865 https://hal.science/hal-01821865}

Submitted on 22 Jun 2018

HAL is a multi-disciplinary open access archive for the deposit and dissemination of scientific research documents, whether they are published or not. The documents may come from teaching and research institutions in France or abroad, or from public or private research centers.
L'archive ouverte pluridisciplinaire HAL, est destinée au dépôt et à la diffusion de documents scientifiques de niveau recherche, publiés ou non, émanant des établissements d'enseignement et de recherche français ou étrangers, des laboratoires publics ou privés. 


\title{
Modelling of the microstructure of mesoporous alumina constrained by morphological simulation of nitrogen porosimetry
}

\author{
Published in Colloids and Surfaces A: Physicochemical and Engineering Aspects \\ Vol. 553, 2018, pp. 378-396
}

DOI: $10.1016 / j \cdot c o l s u r f a .2018 .05 .043$

\author{
Haisheng Wang ${ }^{\mathrm{a}}$, Dominique Jeulin ${ }^{\mathrm{a}}$, François Willot ${ }^{\mathrm{a}}$, Loïc Sorbier ${ }^{\mathrm{b}}$, Maxime Moreaud ${ }^{\mathrm{b}}$ \\ ${ }^{a}$ MINES ParisTech, PSL - Research University, CMM - Centre for Mathematical Morphology, 35, rue St Honoré, F-77300 \\ Fontainebleau, France \\ ${ }^{b}$ IFP Energies Nouvelles, Rond-point de l'échangeur de Solaize, BP 3, 69360 Solaize
}

\begin{abstract}
A procedure is proposed to simulate numerically experimental capillary condensation and evaporation isotherms in porous media, and to simulate random microstructures based on such isotherms. The present methodology is applied to a mesoporous material made of nanometric alumina "platelets". First, a numerical method is developed to compute the adsorption and desorption isotherms in digital porous media. The method, based on simple morphological operators, extends that of Münch and Holzer [15] on mercury porosimetry. The meniscus of the vapour-liquid interface occurring during adsorption are simulated using a morphological closing $[13,17]$ of the solid phase by a sphere. The diameter of the sphere controls the radius of curvature of the vapour-liquid interface. To simulate desorption, a combination of morphological closing and hole-filling operators is used. For random media, the desorption curve is strongly sensitive to the volume of the computational domain. We overcome this effect by a percolation analysis of the gaseous phase during desorption. The present method allows one to predict the hysteresis and pore size distribution associated to porosimetry. To validate this approach, numerical results on simple geometries are compared with the work of Štěpánek et al. [19] based on the Kelvin equation. The condensation and evaporation isotherms occurring in various ideal Boolean models simulating different types of porous media are also computed and interpreted. Second, nitrogen porosimetry data for mesoporous alumina is considered. Based on results obtained for multiscale microstructures, a random model is proposed for mesoporous alumina. To control both the size distribution and hysteresis, the porosity is made of a union of Boolean and hard-core models of spheres. The parameters of the model are identified and numerically adjusted to reproduce the experimental desorption isotherm. Finally, we develop a model of mesoporous alumina, consistent with both porosimetry data and Transmission Electron Microscopy images, made of aggregates of locally-aligned alumina platelets, as introduced in a previously-developed model [23]. The model contains the following characteristic scales: the platelets' size, that of platelets aggregates, and the size-distribution of pores.
\end{abstract}

Keywords: Capillary condensation, Porosimetry, Adsorption, Random model, Pore size distribution, Microstructure modelling

\section{Introduction}

This work focuses on the modelling of the capillary condensation and evaporation phenomena, as described described by the Kelvin equation, in porous media. The Kelvin equation relates vapour/pressure equilibrium to the morphology of the pores: the more curved the local vapour/liquid interface is, the lower the equilibrium vapour pressure is. Accordingly, capillary condensation occurs first along highly curved interfaces. Porosimetry isotherm represents the amount of liquid (or equivalently, the remaining porosity filled by gas) as a function of pressure. When represented as a function of curvature radius, the isotherm is frequently interpreted as a cumulative size distribution for the porous phase [1]. Accordingly, the simulation of the capillary condensation and evaporation is important to study the relation between morphology of the material microstructure and experimental porosimetry data. Nevertheless, the question of determining the main microstructure parameters that influence the porosimetry remains open. 
Štěpánek et al. [19] proposed the method of "virtual capillary condensation" to simulate the nitrogen adsorption and desorption process. The method focuses on the propagation of liquid-vapour interface. At a given temperature and at a given pressure, the Kelvin equation provides a relationship between radius of curvature and pressure. The method measures the local radius of curvature on the solid-vapour or the liquid-vapour interface. Wherever the local radius of curvature is less than the minimum radius, nitrogen liquid is condensed. With liquid condensation and filling, the curved interfaces becomes smooth and less curved. The algorithm converges when the radius of curvature of vapour-liquid interface is equal to the minimum value and the radius of curvature of vapour-solid interface is equal to or larger than the minimum value. At equilibrium, a point in the porosimetry isotherm is obtained. The pressure is then increased, to achieve other equilibrium points in the isotherm. The method is validated for simple geometries like ink-bottle pore, and is also applied to microstructures generated by Gaussian random fields and microstructures of nano-agglomerates and open-cell foams. The pore size distributions of these models are then estimated from the isotherm. Significant computing power is required to simulate the interface propagation on volumes of $100^{3}$ voxels as considered in [19].

In many cases, it is sufficient to compute equilibrium states during nitrogen condensation. These states are obtained experimentally by increasing and decreasing pressure very slowly, so that equilibrium is met at any time. The equilibrium states are governed by the Kelvin equation, which replaces the physical problem by geometrical considerations. In this request, a similar geometrical problem arises in the simulation of Mercury Intrusion Porosimetry (MIP). The analysis of MIP is based on the Washburn equation, which relates the pressure to the radius of intruded cylindrical pores, whose role is similar to the curvature radius in the Kelvin equation. In MIP, mercury intrudes largest pores first, and then, with increasing pressure, intrudes gradually into pores of smaller size. Garboczi and Bentz [8] simulated the MIP in 2D porous structures by the propagation of disc-shape elements from the external borders to the centre of the $2 \mathrm{D}$ space. The disc element of a given radius, which is determined by mercury pressure, occupies the maximum amount of space without overlapping with the solid phase. When the pressure increases, the radius of the disc decreases, and more space is intruded by mercury. Bentz [2] used the method in 3D space and estimated the pore size distribution. At nearly the same time, Thovert et al. [21] proposed the notion of critical sphere in the geometrical characterization of porous media: the largest sphere able to traverse a percolating component. Based on that, Münch and Holzer [15] proposed the notion of "continuous PSD": the amount of pore volume that can be covered by critical spheres. The critical spheres are simulated using double Euclidean distance transforms and thresholds, while the radius of the spheres is equal to the threshold. The "continuous PSD" is then applied to simulate the MIP: despite the "continuous PSD" operation, a regular region growth is performed. The region growth takes the connectivity of the mercury phase into account, and the ink-bottle effect emerges in the simulated intrusion and extrusion curves.

In 1967, Matheron introduced the morphological closing operator - a combination of morphological dilation and erosion, and noted its equivalence with the capillary condensation with spherical structuring element [13]. Münch's method in MIP simulation is similar to Matheron's idea. The double distance transform plus threshold is a specific case of the closing operator when a spherical structuring element is used. Other thermodynamic effects, e.g. solid/liquid interaction [9] and physical multilayer adsorption [4] have been addressed in the literature. Nevertheless, the geometry or the morphology of the porosity dominates the capillary behaviour in meso-pores. For disordered porous media as $\gamma$-alumina, the $3 \mathrm{D}$ porous microstructure is complex, and the representative volume for the capillary behaviour of such disordered porous media is also much larger than ordered porous media. In order to predict the capillary behaviour of a random porous model, numerical simulation methods should be able to handle large volumes to ensure the representativity of the simulated result.

In this work, we propose a novel method that draws on the geometrical MIP simulation described by [15]. It extends to nitrogen porosimetry simulation, in particular, adsorption and percolation analysis. The method is described in Section 2, where simple geometries and a random Boolean model of spheres are considered. The method is then applied to a microstructure model developed for mesoporous alumina (Section 3). A three-scale model of platelets is proposed to reproduce the experimental isotherms, and is validated using TEM images. The limitation of the method is discussed in Section 4. We conclude in Section 5. In Appendix A, we apply the method to various theoretical random sets.

\section{Morphological simulation of nitrogen porosimetry}

Hereafter we consider the Kelvin equation, which reads:

$$
p_{c}=p_{s} \exp \left(-\frac{2 \gamma}{r_{p}} \frac{V_{m}}{R T}\right)
$$


where $p_{c}[\mathrm{~Pa}]$ is the equilibrium vapour pressure above a curved interface, $r_{p}[\mathrm{~m}]$ the local curvature radius of the interface, $\gamma\left[\mathrm{N} \mathrm{m}^{-1}\right]$ the interfacial tension, $R\left[\mathrm{~J} \mathrm{~K}^{-1} \mathrm{~mol}^{-1}\right]$ the universal gas constant, $V_{m}\left[\mathrm{~m}^{3} \mathrm{~mol}^{-1}\right]$ the molar volume of liquid nitrogen, $T[\mathrm{~K}]$ the absolute temperature and $p_{s}[\mathrm{~Pa}]$ is the saturation vapour pressure when the liquid-vapour interface is flat. The Kelvin equation is valid for pores of radius above $2 \mathrm{~nm}$ [20]. Hereafter the curvature radius $r_{p}$ corresponding to the vapour pressure is denoted "Kelvin radius".

Hereafter, the simulation domain $D$, a cuboid of length $l$, is made of a solid phase $S$, represented by its characteristic function:

$$
\chi(x)= \begin{cases}1 & \text { if } x \in S \\ 0 & \text { otherwise }\end{cases}
$$

and a porous phase $S^{c}=D \backslash S$. When the porous media is put into a vapour atmosphere, condensation and evaporation occur and the pore is filled by vapour and condensed liquid. However, not all the pore volume is accessible from the exterior, i.e. there exists pores enclosed by the solid phase. Therefore, before the condensation simulation, a preprocessing is needed to fill isolated pores. We denote the accessible pores by $P$, obtained by a hole-filling morphological operation $H(\cdot)$ so that $P=H(S)^{c}$. The hole-filling operation $H(S)$ is computed by filling all connected components [17] of the porous phase $S^{c}$ unconnected to any border of the domain.

Capillary condensation and evaporation occurs in $P$, more specifically, in areas of a high curvature, e.g. corners and narrow slit pores. During the adsorption process in porosimetry, vapour pressure starts from nearly zero, and increases until the saturation vapour pressure. At any given pressure, an equilibrium between the condensation and the evaporation is established. The criterion of the equilibrium is the radius of curvature, indicated by the Kelvin equation. The maximum radius of curvature of vapour-liquid interface at position $x$ is determined by the radius of the largest sphere in the porous phase containing $x$ [19]. The menisci of condensed liquid-vapour interface is formed along the spheres' boundaries. The filling of areas of high curvature and narrow space and the meniscus can be generated by a morphological operation, a closing by a spherical structuring element:

$$
L\left(r_{p}\right)=\varphi_{r_{p}}(S) \cap P, \quad \varphi_{r}(S)=S \oplus B(r) \ominus B(r),
$$

where $L$ is the liquid phase, $\varphi_{r_{p}}$ a closing operation, $B(r)$ is the spherical structuring element with radius $r$ and $\ominus$ is the Minkowski subtraction (or erosion). In this work, we assume that the system is perfectly wetted and the contact angle is $0^{\circ}$.

A textbook example is provided in Fig. 1: capillary condensation in a conical pore. In a conical pore, the curvature radius decreases linearly in function of pore depth. According to the Kelvin equation, the apex region is filled first with condensed liquid at low pressure. The local curvature radius of liquid-vapour interface and solid-vapour interface should be equal or greater than the Kelvin radius. The closing operation fills the regions of small curvature radius first, and a spherical-cap meniscus is formed on the vapour-liquid interface (Fig. 1a). The curvature radius of every point on the spherical meniscus equals the Kelvin radius. The spherical cap is tangent to the conic solid wall, so the curvature radius at the boundary of the meniscus is also equal or greater than the Kelvin radius. The curvature radius of the remaining vapour-solid interface is greater than the Kelvin radius. Accordingly, the interface resulting from the closing operation satisfies the Kelvin equation, and the capillary condensation phenomenon is simulated.

With increasing pressure, the Kelvin radius increases, the meniscus propagates, and fills the space of large size (Fig. 1b). Even when Kelvin radius is larger than the radius of the cone base, the meniscus becomes the arc of a larger pore outside the conical pore (Fig. 1c). The contact angles are no longer $0^{\circ}$, but the curvature radius becomes negative on the bounary of the arc and still satisfies the Kelvin equation. Another typical example of capillary condensation is the cylindrical pore (Fig. 1d-f) with one side open and the other side closed. Capillary condensation occurs first at the corners, where condensed liquid flatten and smoothen the vapour/liquid interface. The main space of the pores remains empty until the pressure reaches the critical radius, and the pore space is filled all at once.

In practice, approximate spherical structuring elements are used in a discrete grid. The approximations, like rhombicuboctahedron, are not accurate to probe the pore size and to reproduce the menisci. Alternatively, the closing operation with spheres can be realized using Euclidean distance transforms [14] as implemented in [15]. The distance map, denoted by $d_{S}(x), x \in P$, is the distance from a coordinate $x$ to the closest solid-pore interface. The dilation $\delta_{r}(S)$ of the solid phase is obtained simply by a lower threshold:

$$
\delta_{r}(S)=S \oplus B(r)=\left\{x \mid x \in P, d_{S}(x)<r\right\} .
$$



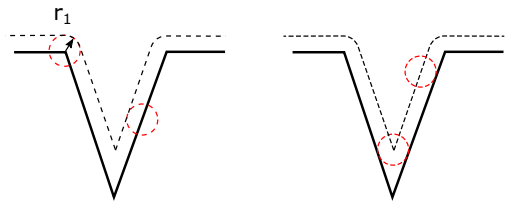

(a)
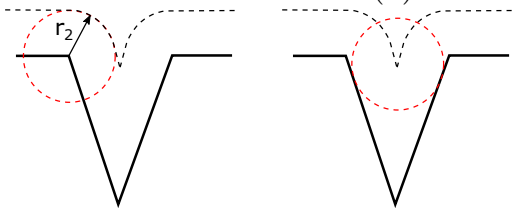

(b)
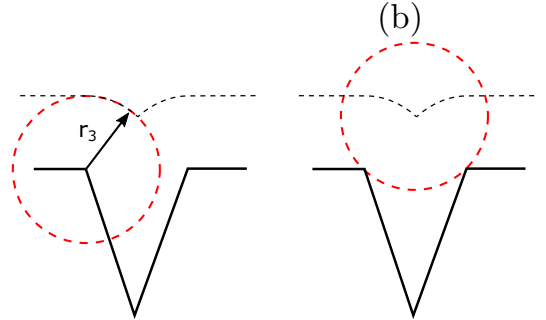

(c)
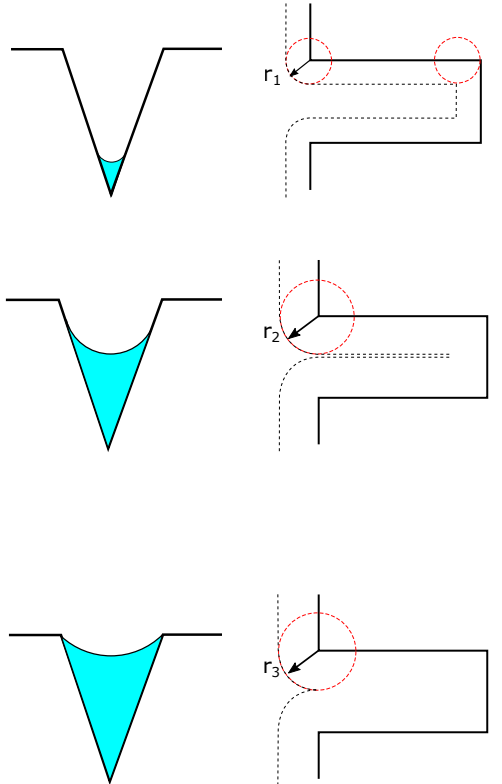

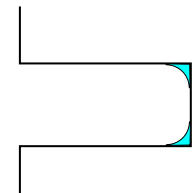

(d)

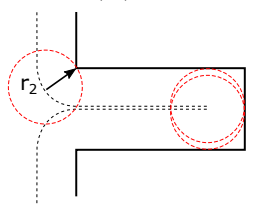

(e)
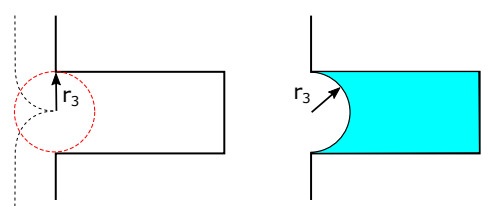

(f)

Figure 1: 2D sections of closing operations in a conical pore (a-c) and cylindrical pore (d-f) (solid black lines). The dilation operation is shown with dotted black line in the left column. The erosion operation is shown in the middle column. The resulting liquid condensation is shown in blue in the right column. Increasing size of the spherical structuring element (dotted red line) are applied (a-b-c and d-e-f).

This transform is followed by an erosion, computed again using the distance function so as to obtain the condensed liquid:

$$
\varphi_{r}(S)=\delta_{r}(S) \ominus B(r)=\left\{x \mid x \in \delta_{r}(S), d_{\delta_{r}(S)^{c}}(x)>r\right\}
$$

The distance map $d_{S}(x)$ for dilation need be calculated once, whereas that for erosion is computed for each value of the radius $r$. After the closing operation, the areas where the curvature radius is inferior to $r_{p}$ are filled. The correct pressure balance is enforced by the spherical opening operation, that leaves interfaces with a given radius of curvature. The Kelvin equation is locally satisfied, and we obtain an equilibrium state in the adsorption process, enforced by the spherical closing operation. The increasing pressure during adsorption corresponds to increasing sphere radius. The procedure is repeated with increasing sphere radius to obtain all equilibrium states.

The volume of condensed liquid at equilibrium state, denoted $\Psi\left(r_{p}\right)$, provides a point on the nitrogen isotherm:

$$
\Psi\left(r_{p}\right)=\mathcal{L}\left(L\left(r_{p}\right)\right)
$$

where $\mathcal{L}(\cdot)$ is the Lebesgue measure. The total volume of adsorbed liquid at saturated pressure is $\Psi_{\infty}=\lim _{r \rightarrow+\infty} \Psi(r)$. In porosimetry isotherm plot, we consider the normalized volume of condensed liquid $\psi$ which depends on the relative pressure (denoted by $p / p_{0}$ ), or the equivalent Kelvin radius:

$$
\psi\left(r_{p}\right)=\Psi\left(r_{p}\right) / \Psi_{\infty}
$$

The continuous pore size distribution, denoted by $I\left(r_{p}\right)$, is given by the normalized intensity of pore volume as a function of pore size. It is evaluated using differentiation [15, 19]:

$$
I\left(r_{p}\right)=\frac{\mathrm{d} \psi\left(r_{p}\right)}{\mathrm{d} r_{p}}
$$

\subsection{Capillary evaporation simulation with morphological opening}

During the adsorption process, each equilibrium state depends only on the pressure and on the solid's morphology, and is independent of previous states of the condensation. However during the desorption process, the evaporation depends on the previous state of condensed liquid. Due to the ink-bottle effect, small "neck pores" block the large pores from evaporation. A blocked large pore remains filled until at least one of the small "neck 


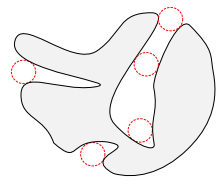

porous media

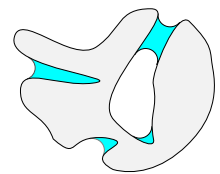

closing

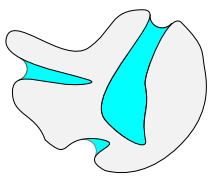

hole-filling

Figure 2: Closing operation followed by a hole filling operation to block the inner pores due to ink-bottle effect.

pores" is released. In practice, when the geometry is disordered and the porous structure is complex, it is difficult to judge the role of "neck pore".

The evaporation occurs only from exterior to interior. It depends both on the local curvature radius and on the connection to the exterior vapour reserve. The following criterion is accordingly considered hereafter: at a given pressure, after the closing operation, if a pore is still connected to the exterior vapour reserve, it is released; if the pore is blocked by condensed liquid or by the solid phase, then it should remain filled. This rule is enforced using a hole-filling operation. Assume that the boundaries of the domain $\partial D$ is the vapour reserve. The operation follows the closing operation, and fills the pores that are not connected to any boundary of the domain. Indeed, this operation eliminates some vapour-liquid interface and some vapour-solid interface around the blocked large pores, and does not change the remaining interface. It is accordingly consistent with the Kelvin equation.

Two steps are therefore used to obtain an equilibrium state during desorption: closing and hole-filling. The liquid phase as a function of sphere radius is given by:

$$
L\left(r_{p}\right)=H\left(\varphi_{r_{p}}(S)\right) \cap P
$$

where $H(\cdot)$ is the hole-filling operator defined by:

$$
H(A)=\left[R_{A^{c}}\left(\partial D \cap A^{c}\right)\right]^{c}
$$

where $R$ is the reconstruction operator. The decreasing pressure during the desorption process corresponds to the decreasing radius of the spherical structuring element (see Fig. 2 for a simulated equilibrium state during evaporation).

The volume filled by the hole-filling operation creates a gap between the adsorption and the desorption branches, and is the origin of hysteresis. The width of the gap is the volume of blocked pores.

\subsection{Multilayer adsorption simulation with morphological dilation}

The phenomenon of physical adsorption of nitrogen molecules on a solid surface is well known. Nitrogen molecules are adsorbed on the solid walls to minimize the interface energy. The molecules also approach the existing molecule layers, and forms multilayer molecules on the solid surface, independent of surface curvature. It happens at low pressures, before the Kelvin equation is valid, but the phenomenon also holds at high pressure. In the BJH method [1] for pore size distribution analysis, the pore size is determined by both the Kelvin radius and the multilayer thickness.

According to the BET theory [4], the number of molecules on the solid interface follows a random distribution. The average number of molecules or layers increases with increasing pressure. We use a dilation operation, denoted $\delta(\cdot)$, with a spherical structuring element to simulate the average thickness of the layers, before the simulation of capillary condensation:

$$
L_{r_{t}}=\delta_{r_{t}}(S) \cap P
$$

where $L_{r_{t}}$ is the multilayer nitrogen molecules, $S$ is the solid phase, $P$ is the accessible porosity and $r_{t}$ is the size of the structuring element, corresponding to the thickness of the multilayer adsorption. In practice, the multilayer adsorption is thin, and the spherical structuring element is small and discretized. The spherical structuring element can be replaced by a 3D cross or by the Euclidean distance transform followed by a threshold. The later option is more accurate and used in the present work.

Multilayer adsorption is followed by capillary condensation. We make use of the dilated microstructure $S \cup L_{r_{t}}$ to simulate it. The liquid phase in the microstructure is then obtained by the closing operator:

$$
L\left(r_{p}\right)=\varphi_{r_{p}}\left(S \cup L_{r_{t}}\right) \cap P .
$$




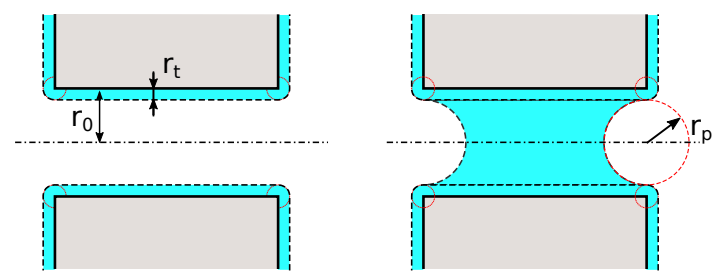

(a)

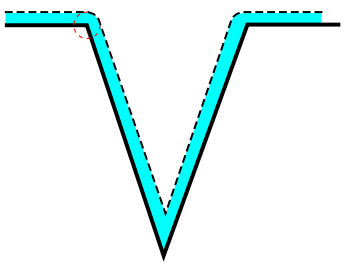

(b)

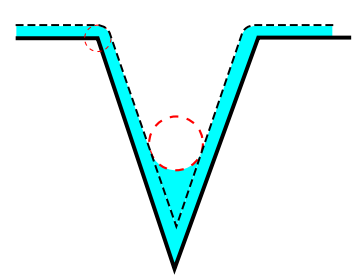

Figure 3: 2D sections of cylindrical (a) and conical (b) pores with multilayer adsorption (left images) and capillary condensation (right images). Solid phase is in grey. Liquid phase is in blue. Red dotted lines are the structuring elements.

Note that capillary condensation simulation should not start from a zero Kelvin radius. This is because at low pressure, the thermodynamic multilayer adsorption, not the capillary condensation, dominates the volume of condensed liquid. The Kelvin equation is valid for a Kelvin radius larger than $2 \mathrm{~nm}$ [20]. The domain of Eq. 12 is accordingly set to be $r_{p} \geq 2 \mathrm{~nm}$. The local pore size, denoted $r$, is then determined by both the multilayer thickness and the Kelvin radius:

$$
r=r_{t}+r_{p}
$$

The simulation method by dilation is illustrated for a cylindrical pore with base radius $r_{0}$ and two open ends in Fig. 3a. At low pressure, only the multilayer of molecules of thickness $r_{t}$ is adsorbed on the solid interface. With increasing pressure, when the Kelvin radius satisfies $r_{p}=r_{0}-r_{t}$, the pore is filled and the meniscus is formed at the two ends. The boundaries of the meniscus are tangent to the existing liquid interface. The newly formed vapour/liquid interface is smooth and satisfies the Kelvin equation. Another schematic illustration of the multilayer adsorption simulation in a conical pore is shown in Fig. 3b. After multilayer adsorption on the cone surface, the capillary condensation is simulated with a spherical structuring element of radius 2 nm, instead of radius starting from $0 \mathrm{~nm}$.

To implement this method, the multilayer thickness $r_{t}$ at low pressure needs to be identified. According to [11], the thickness of a nitrogen monolayer is $0.354 \mathrm{~nm}$. More precisely, one needs to know how many layers are present before capillary condensation occurs at $r_{p}=2 \mathrm{~nm}$. According to the Kelvin equation, the Kelvin radius $r_{p}=2$ $\mathrm{nm}$ corresponds to relative pressure $p / p_{0} \approx 0.6$. As mentioned in the experimental isotherms, the volume fraction of condensed liquid in the porosity at $p / p_{0}=0.6$. This value corresponds to the volume fraction of the multilayer adsorption and the initial volume fraction by capillary condensation at $r_{p}=2 \mathrm{~nm}$. With the BET equation, we obtain the gas quantity adsorbed in a monolayer nitrogen, which takes a volume fraction of the porosity of $8.24 \%$. The proportion $23 \% / 8.24 \%$, which indicates that the average number of adsorbed layers at $p / p_{0}=0.6$, is strictly below 3. Thus, in the multilayer adsorption simulation by dilation, the thickness $r_{t}$ is either 1 or 2 layers. This question is addressed in the following section.

\subsection{Ink-bottle pore}

An ink-bottle pore consists of two embedded cylindrical pores having the same centre axis (Fig. 4). The diameters and heights of the cylinders are measured from the figures in [19]. The radius of the outer cylinder, denoted by $r_{1}$, is 50 voxels and that of the inner pore, denoted by $r_{2}$, is 100 voxels. We apply the capillary condensation simulation on the ink-bottle pore (see 3D view, Fig. 4) and obtain the condensation isotherm represented in Fig. 5.

The condensation occurs first at the corners of the inner pore, when the Kelvin radius $r_{p}$ is lower than the radius of the outer cylinder pore $r_{1}$ (Fig. 4a). When the Kelvin radius is larger than $r_{1}$, the outer pore is filled by condensed liquid, and the hemispherical menisci is formed with sphere radius $r_{1}$ (Fig. 4b). Condensation occurs in the inner pore with increasing curvature radius, until it reaches the radius of the maximum sphere $r_{\text {max }}$ that can fit in the pore (Fig. 4c). Note that the $r_{\max }$ is less than $r_{2}$ because the inner pore is not deep enough to fit a larger sphere.

The Kelvin radius $r_{p}$ decreases during evaporation. When $r_{p}$ is inferior to the maximum sphere radius $r_{m a x}$, the inner pore would not be released because it is not connected to the outer vapour reserve. Vapourization occurs solely on the surface of the outer pore. The radius of the menisci decreases (Fig. 4e), until it reaches $r_{1}$. The outer cylindrical pore is then released, together with the inner pore (Fig. 4f). Some condensed liquid remains at the corners of the inner pore. The latter is vaporized with decreasing pressure.

Our results for the capillary condensation/evaporation simulation on slit pore and ink-bottle pore are qualitatively consistent with that of [10] obtained by density functional theory. No quantitative data is available in the later 


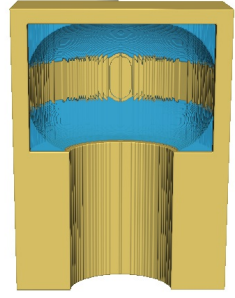

(a)

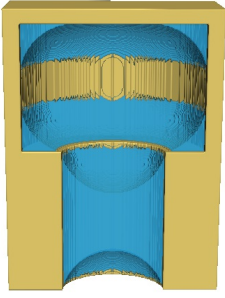

(b)

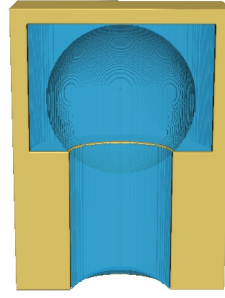

(c)

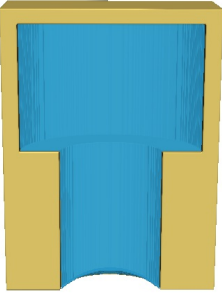

(d)

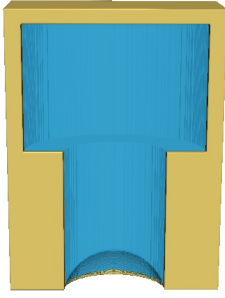

(e)

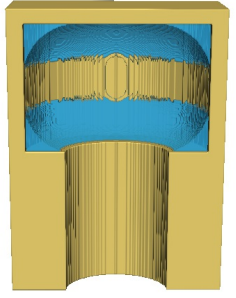

(f)

Figure 4: Ink-bottle pore with condensed liquid in blue and solid in yellow. The images from (a-d) are equilibrium states during adsorption at increasing pressure. Images (e) and (f) are equilibrium states during desorption at decreasing pressure. The pressures are marked in the Fig. 5a-e.

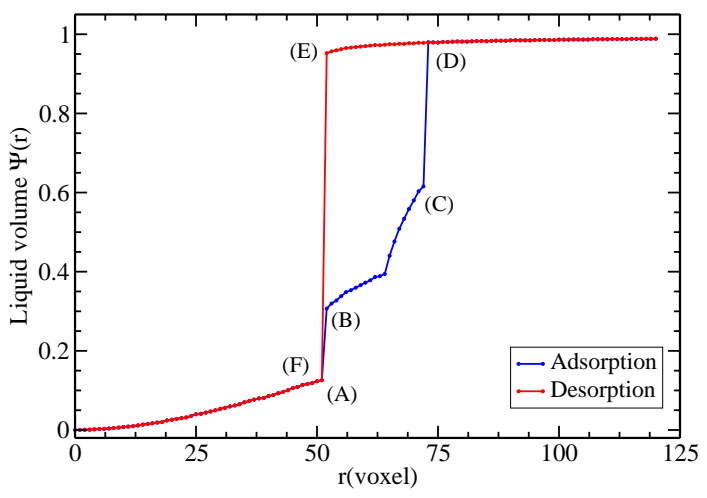

Figure 5: Nitrogen adsorption-desorption hysteresis curve simulated on the ink-bottle pore by our approach. The shape of the liquidvapour interface of each marked pressure point is represented in Fig. 4a-e.

reference. The predicted isotherms are also close to that obtained by virtual capillary condensation method [19], in particular the filling pressure of the inner pore (Fig. 5 points $\mathrm{C}$ to $\mathrm{D}$ ), the release pressure of the inner pore (Fig. 5, point $\mathrm{E}$ to $\mathrm{F}$ ). The variation of the pressure when the outer pore is filled (Fig. 5 points A to B) is however different in the two approaches. This is because the two methods predict two different local minima. Both the hemispherical vapour/liquid interface produced by our method and the cylindrical vapour/solid interface predicted by the virtual condensation method satisfy the Kelvin equation. The hypothesis of equilibrium during adsorption is nevertheless questionable (see $[3,16]$ ). Our simulation on the ink-bottle pore is also consistent with the results obtained by DFT and Monte Carlo simulation [10].

\subsection{Validation of the method on cylinder-sphere grid pore}

Consider now a regular porous network made of 8 spherical pores connected by cylindrical pores connected to the outer space (Fig. 6a), resulting in a porous medium with strong ink-bottle effect. The radii of the cylindrical and spherical pores take on values $r_{c}=24$ and $r_{s}=27$ voxels respectively. The pore size distribution of such structure results, in theory, into two peaks. During adsorption, the capillary condensation does not occur when the Kelvin radius $r_{p}$ is less than $r_{c}$. When $r_{s}>r_{p} \geq r_{c}$, the cylindrical pores are filled (Fig. 6b-c), whereas, when $r_{p} \geq r_{s}$, the entire porous network is filled by condensed liquid (Fig. 6d). In the simulated isotherm in Fig. 7, we see two steps, corresponding to the two pore sizes. The ink-bottle effect is also reproduced in this regular pore grid - the liquid in the spherical pores are released only after the release of the cylindrical pores.

\subsection{Boolean model of spheres}

Consider as a random isotropic structure the Boolean model of equisized spheres, obtained by an underlying homogeneous Poisson point process for the sphere centres. The pores are the complementary set of the union of spheres, so that their shapes and sizes can be seen as random as compared to previous geometries. Spheres with diameter 50 voxels are used to generate a porous medium with $50 \%$ volume fraction, discretized on grids of $256 \times 256 \times 256$ volume in voxels. Condensation and evaporation are illustrated in Fig. 8 (maps a-d and e-h, respectively) whereas the predicted isotherm is in Fig. 9. 


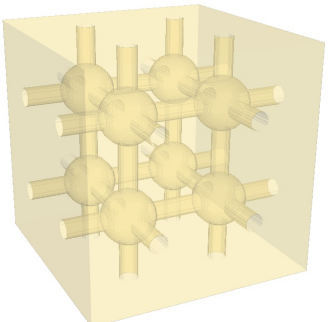

(a)

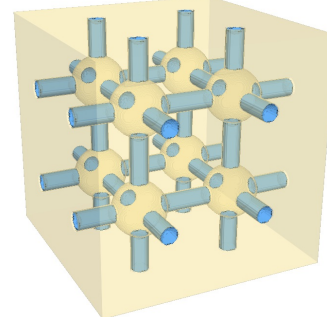

(b)

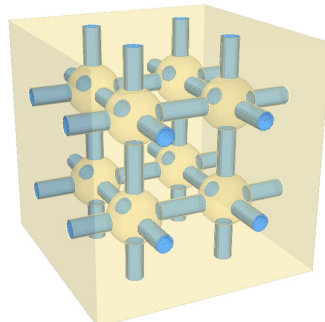

(c)

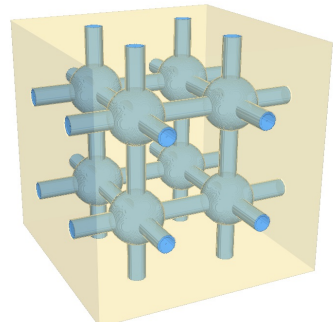

(d)

Figure 6: Condensation in a regular cylinder-sphere grid pore.

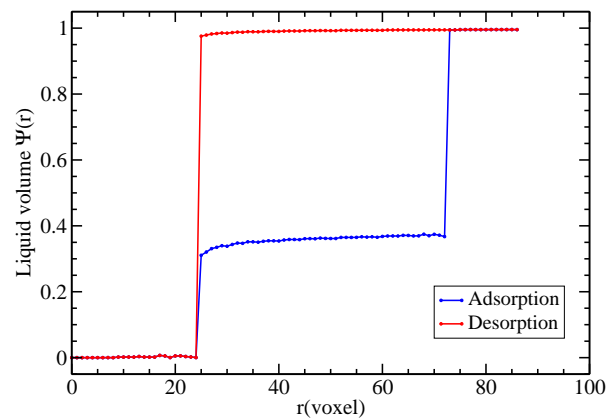

Figure 7: Capillary condensation/evaporation isotherm simulated on the regular pore grid.

Condensation (Fig. 8, white zones) first occurs at the corners of intersections and in the narrow space between two spheres. Small pores are filled as the Kelvin radius increases (Fig. 8b), and vapour "bubbles" are formed inside the microstructure (Fig. 8c). When the pressure is high enough, the Kelvin radius is sufficiently large for the condensed liquid to fill the whole porosity. During the desorption process, large pores at the boundary that are connected to the exterior vapour reserve are released first (Fig. 8e-h). Because of the ink-bottle effect, some large pores are blocked inside from releasing. Note that the 2D sections cannot correctly present the real curvature of the meniscus; we have verified that all liquid-vapour interfaces in $3 \mathrm{D}$ have equal curvature (not shown).

As shown in the isotherm (Fig. 9, radius $R=40$ ), nearly no hysteresis is obtained for the Boolean model with equisized spheres. The other curves correspond to Boolean model of equisized spheres discretized on the same grid of voxels, with the same volume fraction, but with radii $R=20$ and 60 voxels. A slight hysteresis is observed when the spheres are smallest, i.e. when the number of spheres is highest. This difference is due to the relative representativity of the structures, or to the finite-size effect of the domain, and is discussed hereafter.

\subsection{Sensitivity of the isotherms with respect to representativity and resolution}

The hysteresis in sorption isotherm should be related to the amount of large pores that are not well-connected to the boundary of the volume. The system size plays an important role in determining the connection of the porous network to the exterior vapour reserve. To study the boundary effect of the microstructure on the isotherms, we consider three realizations of Boolean models of obstacle spheres of radius 20 voxels, in volumes containing $256^{3}$, $512^{3}$ and $1024^{3}$ voxels (Fig. 10a). The number of spheres in the three microstructures increase by a factor of 8 . As expected, the hysteresis increases when the number of spheres increases, i.e. when the volume is more representative of a macroscopic sized mesoporous sample. Furthermore, as expected, the desorption branch is also smoother when the microstructure is more representative. The difference is amplified for Boolean models of spheres of radius 10 voxels (Fig. 10b). Compared to the desorption branches, the adsorption branches are much less sensitive to the system size. The adsorption branches converge to an asymptotic curve when the number of spheres becomes very large (Fig. 10b), however this convergence is very slow for desorption.

The effect of resolution is studied on a Boolean model of spheres of diameter 40 voxels. A microstructure is first simulated in a volume containing $1024^{3}$ voxels. Downsampling is performed on the microstructure, with decimation factors of 2 and 4 . The capillary condensation and vapourization simulation isotherms are shown in Fig. 11. The horizontal axis of the isotherms of the downsampled microstructures have been corrected by multiplying by their 


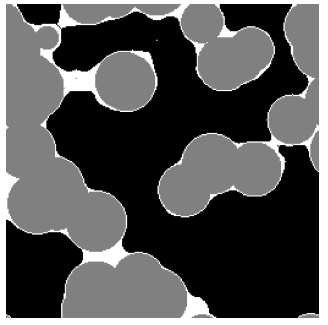

(a)

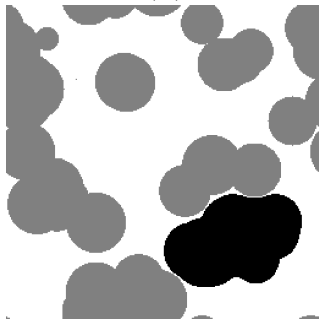

(e)

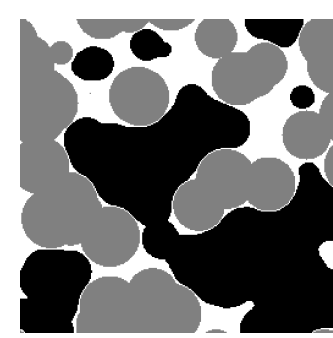

(b)

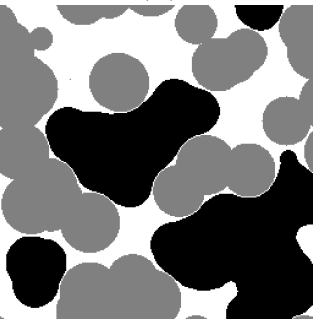

(f)

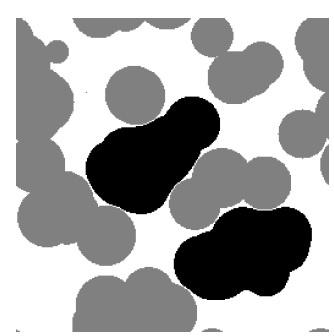

(c)

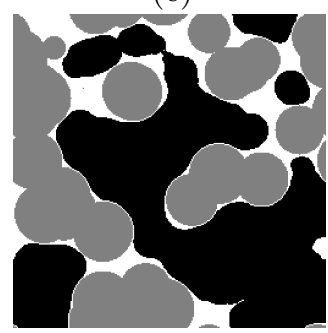

(g)

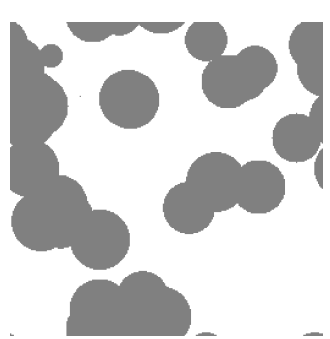

(d)

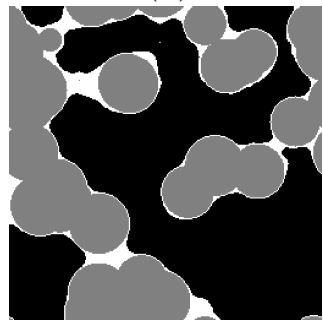

(h)

Figure 8: 2D sections of equilibrium states during the capillary condensation (a-d) for increasing radii and evaporation (e-h) for decreasing radii in a 3D Boolean model of solid spheres. Vapour phase is in black, solid phase is in grey and liquid phase is in white.

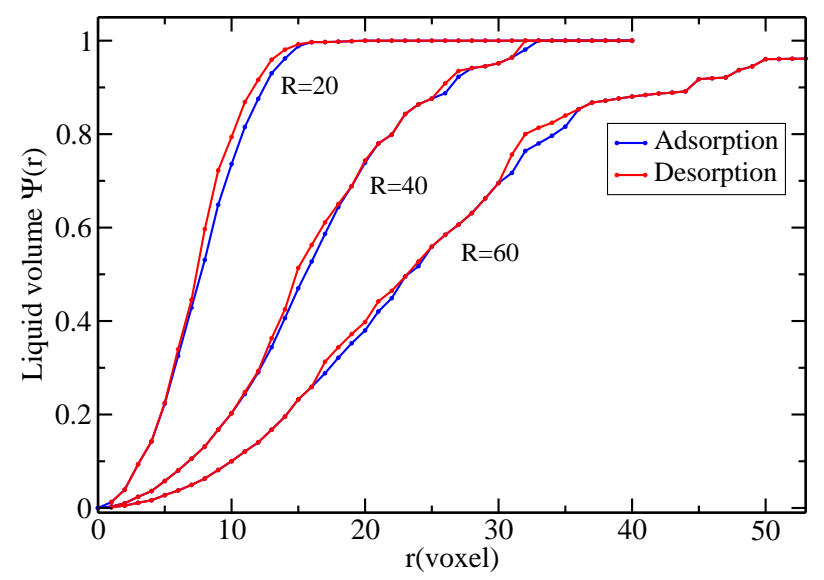

Figure 9: Capillary condensation (blue) and evaporation (red) isotherms of Boolean models of spheres, of increasing radius $R=60$ voxels, $R=40$ voxels and $R=20$ voxels (bottom to top). 


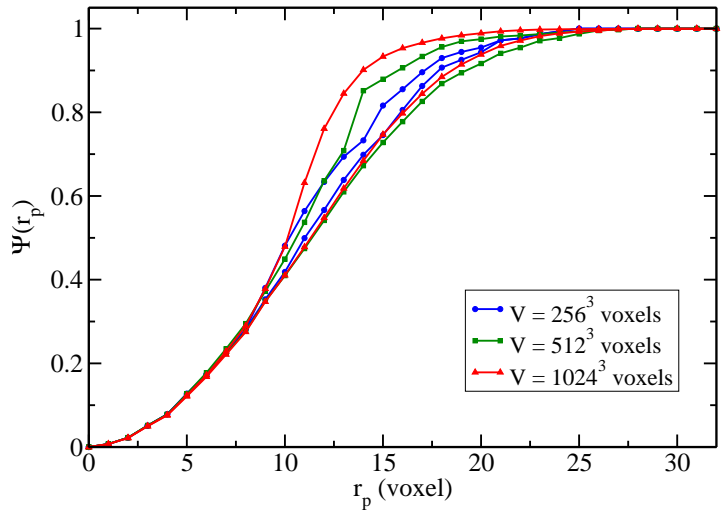

(a)

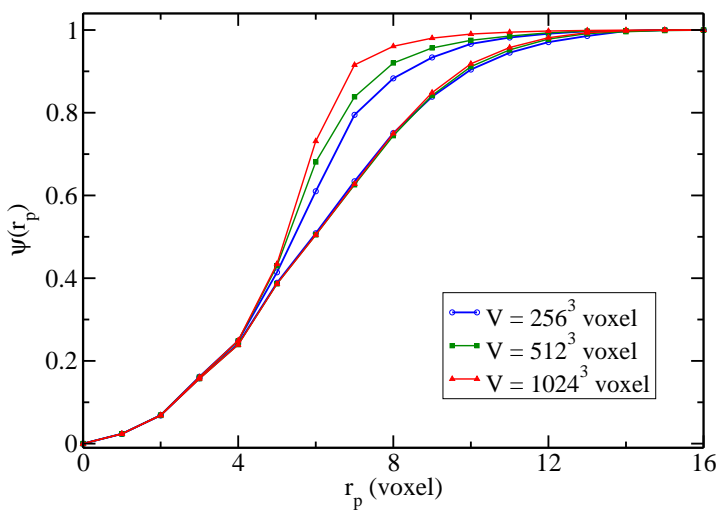

(b)

Figure 10: Effect of representativity of the domain: capillary condensation isotherm in three Boolean models of spheres of different sizes. The volume fraction of solid phase is 0.65 . (a) The sphere radius is 20 voxels. The number of spheres are $525\left(V=256^{3}\right.$ voxels), $4205\left(V=512^{3}\right)$ and $33639\left(V=1024^{3}\right)$. (b) The sphere radius is 10 voxels. The number of spheres are 4205 (for $V=256^{3}$ voxels), 33639 (for $V=512^{3}$ voxels) and about $2.7 \times 10^{5}$ (for $V=1024^{3}$ voxels).

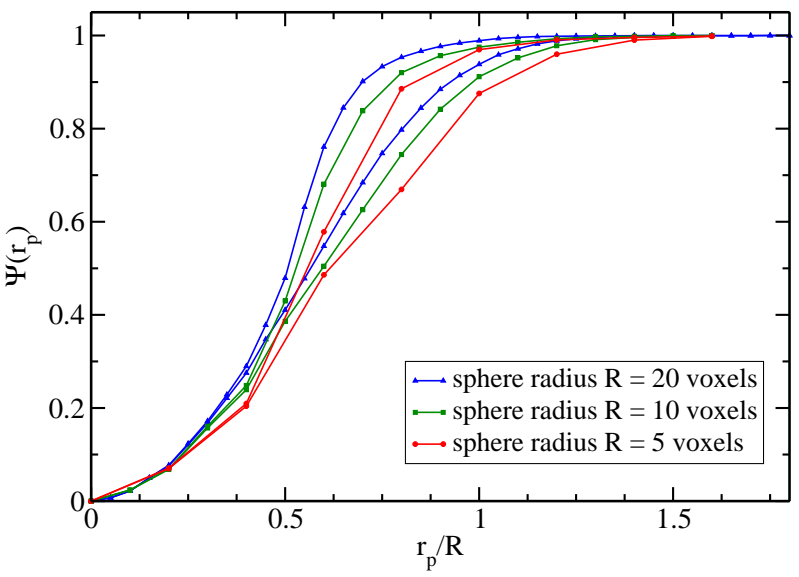

Figure 11: Effect of discretization: capillary condensation isotherm in a Boolean models of spheres at coarse, intermediate and fine resolutions.

decimation factors. As shown in Fig. 11, at low resolution, the adsorption branches and the desorption branches are both less smooth and are both shifted to the right, resulting in an overestimation of the pore size. The simulated isotherms are accordingly quite sensitive both to the representativity of the system (volume size with respect to the spheres radius) and to the resolution (radius in voxels). Changes of the boundary condition, such as the use of a surface or a point where vapour is injected, or the use of periodic boundary conditions, were considered. The later are insufficient to overcome the effect of representativity and another, computationaly-efficient method, is sought for.

In porosimetry, the pores which are directly connected to the exterior will be released once the local curvature radius satisfies the Kelvin equation. However, most pores will remain filled because of the ink-bottle effect. The proportion of the released exterior pores tends to 0 when the system volume tends to infinity, which is the case in real experiments. In the simulations, due to finite-size effect, the desorption branch does not remain constant and equal to 1 , but decreases along with the pressure decreasing. We identify the pressure value when the desorption branch reaches the desorption threshold of the vapour phase. Thus in capillary evaporation simulation, we should consider the percolation of the vapour phase as the point where desorption occures. Use of such threshold is consistent with experimental isotherms (Fig. 13), or typical type-IV isotherm in porosimetry (see [18]) where the desorption branches remains constant and equal to 1, until the decreasing pressure reaches a threshold. The same phenomenon has been observed in other works, e.g. [12].

We accordingly check for the percolation of the vapour phase at each equilibrium state. If there exists a connected 


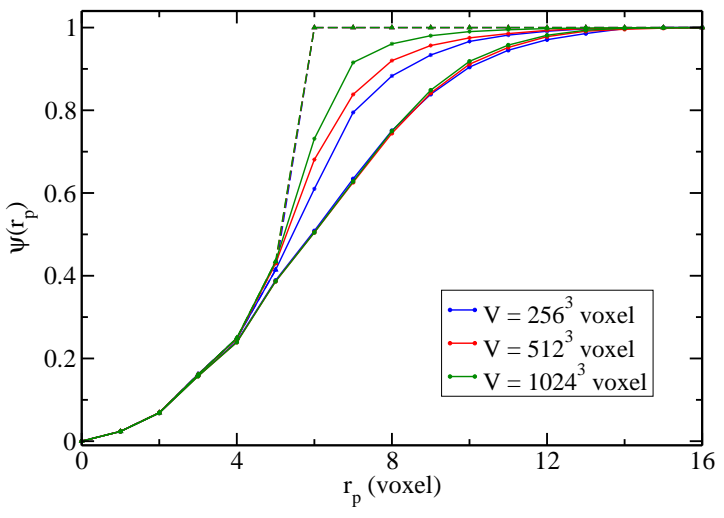

(a)

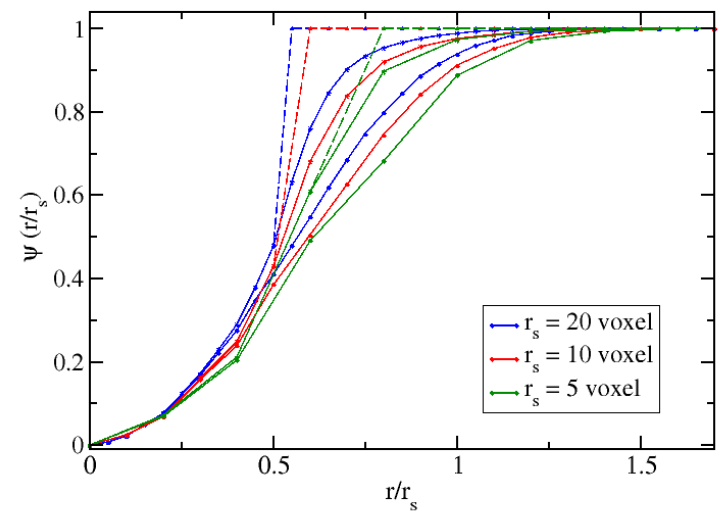

(b)

Figure 12: Capillary condensation/evaporation isotherms with percolation correction of Boolean models of spheres with $65 \%$ volume fraction for the solid phase. (a) Representativity effect. Different system sizes and constant sphere radius 10 voxels. (b) Discretization effect. Identical numbers of spheres (33639) but different sphere radii.

component of the vapour phase which reaches the 6 faces of the cube, the vapour phase percolates. Although other percolation criteria can be used, no other has been explored in the present work. Using this onset of percolation as threshold, the desorption branch is adjusted to simulate the evaporation in a system of infinite or very large size as follows:

$$
\psi^{\prime}(r)= \begin{cases}\psi(r), & \text { if } \exists j, \forall i \in\{1,2, \ldots, 6\},\left\{C_{G}(r)\right\}_{j} \cap(\partial D)_{i} \neq \emptyset \\ 1, & \text { otherwise }\end{cases}
$$

where $\psi^{\prime}$ is the normalized volume of condensed liquid for an infinite-size system, $(\partial D)_{i}, i=1,2, \ldots, 6$ are the six faces of the cubic domain and $C_{G}(r)$ is the set of labeled connected components of the vapour phase $G(r)=\left\{P^{c} \cup L(r)\right\}^{c}$.

For comparison, we take the same Boolean microstructures as studied in Fig. 10, and compute the corrected desorption branch using percolation (Eq. 14). The desorption branches of infinite-sized systems are shown in dotted lines. Figure 12a shows that, once the percolation is taken into account, the desorption threshold of the vapour phase in a Boolean model of spheres varies little with respect to microstructure size.

Fig. 12b shows the effect of discretization. For a fixed number of spheres, a coarse resolution leads to an overestimation of the desorption threshold. The isotherms obtained with the two higher resolution are close to each other. Smoother curves are obtained for the highest resolution. These results suggest that an object with near spherical shape should be discretized on a grid with voxel size not larger than $1 / 40$ times the lnegth of the object's diameter.

The numerical method described in this section to simulate the nitrogen porosimetry hysteresis can be decomposed into multilayer adsorption by dilation, capillary condensation by closing, capillary evaporation by opening, combined with desorption threshold probing by connected components, and multilayer desorption by erosion. Most of the CPU time and memory required to carry out the computations is spent by the distance transform. We use the fast algorithm in [14] to compute an exact Euclidean distance transform. A pair of equilibrium states, respectively in the adsorption branch and in the desorption branch at the same Kelvin radius takes 3 minutes for a system of $1024^{3}$ voxels (with 24-nodes parallelization on CPU of $2.67 \mathrm{GHz}$ ).

\section{Application to microstructure modelling of mesoporous alumina}

\subsection{Nitrogen porosimetry measurement}

A mesoporous alumina sample under study was synthesized from commercial boehmite powders. The detailed synthesis process is described in [23]. It has been characterized by nitrogen porosimetry (see Fig. 13a) which follows typical type-IV isotherms [18] with gradual adsorption branch and steep desorption branch.

The experimental isotherms contain information on multilayer adsorption, capillary condensation and liquid compressing. To highlight the capillary condensation and evaporation part, we use the method proposed by [6] to 


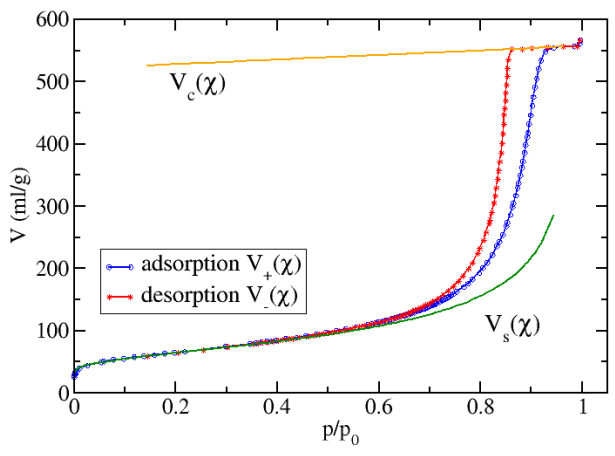

(a)

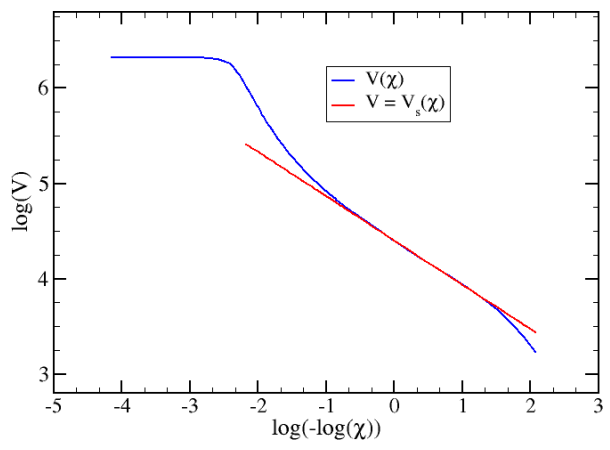

(b)

Figure 13: Fit of the adsorption branch in nitrogen porosimetry by Eq. 16 .

preprocess the isotherms. The method removes the effects of liquid compressibility at high pressure with a linear fit, and removes the effects of multilayer nitrogen molecule adsorption at low pressure with the FHH equation. The principles of the method are as follows. First, a linear fit of the high pressure part of the isotherm is defined, denoted $V_{c}(\chi) \propto \chi$, where $\chi=p / p_{0}$ is the relative pressure. Second, the low pressure part is defined as $V_{s}(\chi)=S \cdot h(\chi)$, where $S$ is the specific surface area, and $h(\chi)$ is the thickness of the multilayer molecule film, usually modelled by the FHH equation [6]

$$
h(\chi)=\left[\frac{K}{-\log \chi}\right]^{1 / m}
$$

The prefactor $K$ and exponent $m$ are constants related to the chemical nature of the nitrogen-alumina interface. The equation is identified by curve fitting for each isotherm. Eq. 15 is rewritten as:

$$
\log V_{s}(\chi)=-\frac{1}{m} \log (-\log \chi)+\log S+\frac{1}{m} \log K
$$

We use Eq. 16 to fit the linear part of the original experimental isotherms (denoted $V(\chi)$ shown in Fig. 13 B). Therefore, the FHH equation $V_{s}(\chi)$ with identified parameters is obtained:

$$
-\frac{1}{m}=-0.465, \quad \log S+\frac{1}{m} \log K=0.402
$$

The result in Eq. 17 is sufficient to compute $V_{s}(\chi)$ with Eq. 16, without knowing the exact values of $K$ and $m$. The linear curve fit result is shown in Fig. 13. Furthermore, in the rest of this work, we denote the filtered and normalized isotherms by:

$$
Q(\chi)=\frac{V_{c}(\chi)-V(\chi)}{V_{c}(\chi)-V_{s}(\chi)}
$$

The pore size distribution $I(r)$ defined in Eq. 8 is estimated from the normalized isotherm $Q(r)$ and shown in Fig. 14.

\subsection{Reminder: microstructures of mesoporous alumina}

Two microstructures have been introduced in previous works to simulate the alumina sample. The first one is a tomographic reconstruction [22] (Fig. 15). A few calcined extrudates were crushed in a mortar and the obtained powder was dispersed in ethanol. A drop of the suspension was put on a holey carbon grid that was dried. Tilt series projections were acquired on a JEOL JEM-2100F fitted with the Gatan Ultrascan CCD camera, in bright field mode at $200 \mathrm{kV}$. 143 projections with an indicated magnification of 40000 times $(0.21 \mathrm{~nm}$ pixel size $)$ were acquired at full camera resolution (2048 by 2048 pixels). The 143 tilt angles ranged between $-71^{\circ}$ to $+71^{\circ}$ with a Saxton spacing scheme. No fiducial marker was put on the grid to avoid the artifacts induced in their vicinity. Prior to reconstruction, images were binned twice to obtain a 1024 by 1024 pixels tilt series. The 3D volume was reconstructed with the robust method based on inverse problem approach to align marker-free projections and reconstruct $3 \mathrm{D}$ volume [22]. 


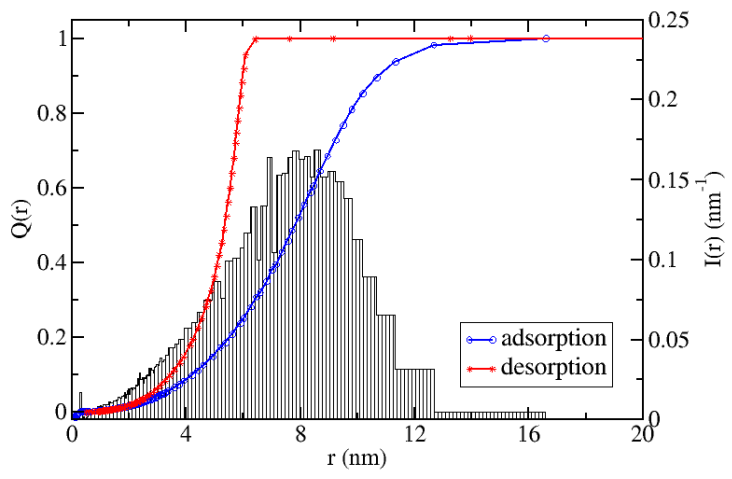

Figure 14: Filled volume fraction deduced from nitrogen porosity isotherms and pore size distribution estimated from Eq. 8.

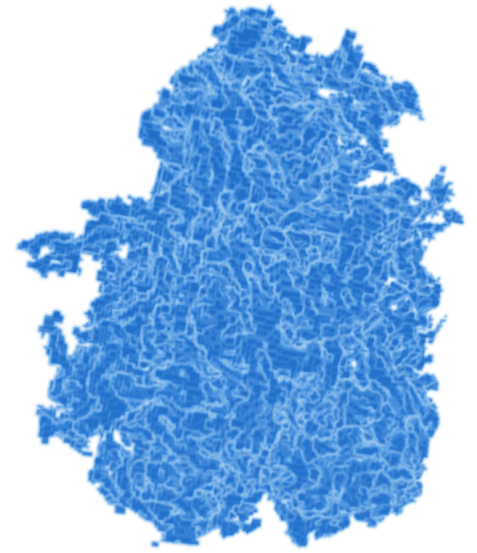

Figure 15: 3D view of the tomographic reconstruction of a small portion of mesoporous alumina. The image has a $0.87 \mathrm{~nm}$ voxel size, and the image field of view is $328 \mathrm{~nm} \times 290 \mathrm{~nm} \times 257 \mathrm{~nm}$.

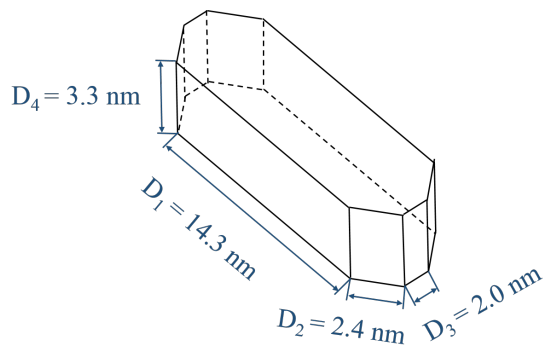

Figure 16: Prism shape of $\gamma$-alumina grain identified with the TEM images of the mesoporous alumina sample. 


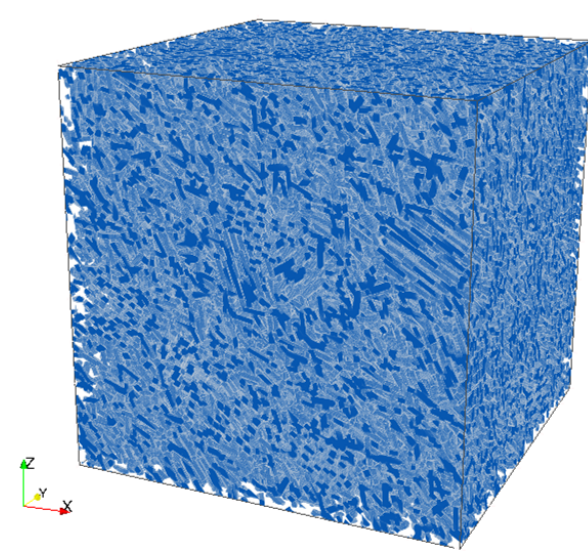

(a)

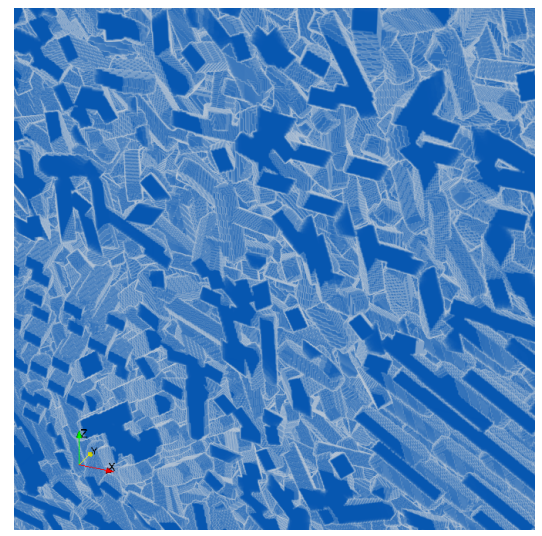

(b)

Figure 17: 3D simulation of the microstructure of mesoporous alumina with the two-scale model of platelets identified with TEM images and specific surface area. The total number of implanted platelets in the microstructure is 63506, including 12141 aligned platelets and 51365 randomly-oriented platelets. The parameters of the model is shown in Table 1 (column "platelets" and column "aggregates"). (a) Size $300 \times 300 \times 300 \mathrm{~nm}^{3}$. (b) Enlargement of a $2 \mathrm{D}$ cut of size $50 \times 50 \mathrm{~nm}^{2}$.

The second one is a two-scale random model of platelets $[23,24]$ based on TEM images of mesoporous alumina, and generated by following procedure: (i) a Boolean model of spheres of fixed diameter, each sphere assigned with a random orientation uniformly sampled on the unit sphere; (ii) two Poisson point processes with two different densities are generated inside and outside the spheres; (iii) a platelet with a fixed shape is located at each Poisson point. The platelet has a random direction if the point is outside the sphere. It has the direction assigned to the sphere if the point is inside a sphere. If the point belongs to several spheres, the platelet direction is chosen randomly between the directions assigned to the spheres. Such a model has eight parameters, four defining the shape of the platelets (Fig. 16) and four controlling the aggregation state: the sphere radius $r_{\mathbf{S}}$, the volume fraction of spheres $p_{\mathbf{S}}$, the volume fraction of platelets in the spheres $p_{\mathbf{A}}$ and the volume fraction of platelets outside the spheres $p_{\mathbf{B}}$. The four latter parameters are related with the porosity $\varepsilon$ by:

$$
p_{\mathbf{B}}\left(1-p_{\mathbf{S}}\right)+p_{\mathbf{A}} p_{\mathbf{S}} \approx 1-\varepsilon
$$

Parameters of the model were identified from TEM images of sliced sample by an inverse problem approach with L2-norm minimization of the correlation function [23]. The parameters are re-identified with high frequency noise taken into account [24] and are shown in Table 1. The platelet size and shape are in agreement with TEM image observations and with literature [5]. The model is validated by specific surface area measured by nitrogen porosimetry. A 3D realization of the identified two-scale model is shown in Fig. 17.

\subsection{Capillary condensation simulation of a tomographic reconstruction}

The reconstructed 3D image size is $328 \mathrm{~nm} \times 290 \mathrm{~nm} \times 257 \mathrm{~nm}$ at resolution $0.66 \mathrm{~nm}^{3}$ per voxel. The capillary condensation and evaporation simulation were performed on the microstructure, and 2D sections are shown in Fig. 18. The morphological simulation method is processed directly on the segmented 3D tomographic volume. The condensation occurs first on the interface of small curvature radius and in the narrow space between solid walls. The meniscus then propagates gradually from the small pores to large pores, until the whole porosity is filled by condensed liquid.

Simulated isotherms are shown in Fig. 20 in red. The adsorption branch increases slowly until $r_{p}=17 \mathrm{~nm}$, which means a smooth pore size distribution. However, it is observed on the 2D sections that some of the pores larger than $13 \mathrm{~nm}$, are very close to, or directly part of the contour of the sample. It is possible that some of these pores were formed during the sample crushing by mechanical damage. The weak hysteresis means a good connection of the pore phase to the exterior, because of the small system volume and the irregular shape. For the same reason, it is impossible to obtain any information about the desorption threshold. The tomographic reconstruction is not representative enough to simulate the capillary condensation and evaporation behaviour. 


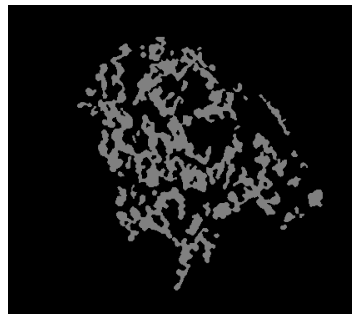

(a)

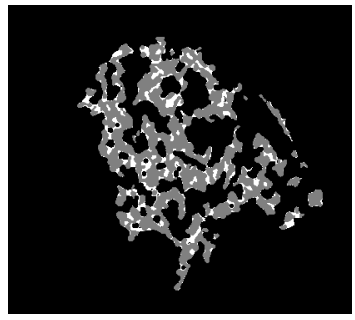

(b)

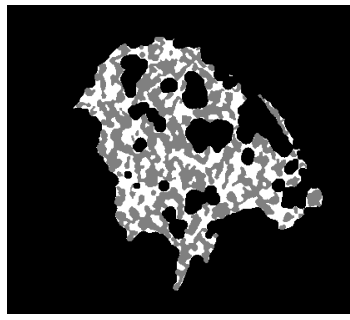

(c)

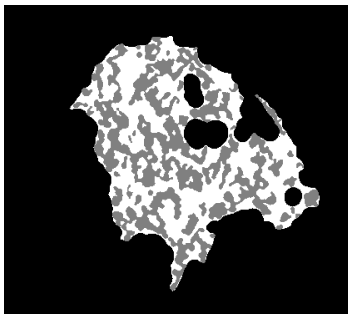

(d)

Figure 18: Capillary condensation simulation on the tomographic reconstruction of a mesoporous sample (2D sections). (a) $r_{p}=0 \mathrm{~nm}$, (b) $r_{p}=3.5 \mathrm{~nm}$, (c) $r_{p}=7 \mathrm{~nm}$ and (d) $r_{p}=10.5 \mathrm{~nm}$.

\subsection{Capillary condensation simulation of a two-scale model of platelets}

We perform the capillary condensation and evaporation on a $1024^{3}$-voxels realization of the identified model (see 2D section, Fig. 19). A compromise is made between spatial resolution, representativity of the domain and computational capacity. The capillary condensation occurs first in the concave corners of intersected platelets. Then the narrow slit space between aligned pores are filled by condensed liquid. The meniscus propagates gradually from small pores to large pores. The large pores locates usually between the aggregates (black zones in Fig. 19b). When the Kelvin radius reaches $10 \mathrm{~nm}$, the whole pore phase is filled.

During the desorption, the pore phase does not percolate until the Kelvin radius reaches $5 \mathrm{~nm}$ and lower. In Fig. 19f, only pores at the boundaries are released. The ink-bottle effect prevents the inner pores from releasing. If we compare the $2 \mathrm{D}$ sections in Figs. $19 \mathrm{~b}$ and $19 \mathrm{e}$, when the pore phase percolates at $r_{p}=5 \mathrm{~nm}$, there are still pores that are blocked by the "neck pores", especially the pores surrounded by aggregates.

The isotherms of the two-scale model is shown in green in Fig. 20. Compared to the experimental isotherms, its pore size distribution is very narrow with smaller average pore size. Indeed, the porosity in the identified two-scale model consists of small regions located in-between platelets, rather than aggregates of platelets. The pore size distribution inside the aggregates and in the complementary is rather the same. Virtual experiments have been performed to study the effects of two scales on the shape of the adsorption/desorption isotherms (Appendix A.4). For mesoporous alumina, the effect of platelet alignment is weak (not presented).

\subsection{Three-scale model of platelets}

\subsubsection{Modelling}

The tomographic reconstruction and the two-scale model of platelets of mesoporous alumina cannot reproduce the experimental isotherms. A microstructure with larger pores and stronger ink-bottle effect is considered in this subsection. We propose to add another scale of exclusions into the two-scale model of platelets. The new model has features of larger pores and better connectivity in the porosity. A three-scale model is constructed in the following way. We first consider a multi-scale model of spherical exclusions to mimics the large pores in mesoporous alumina:

$$
H=\bigcap_{j=m+1}^{n} R_{j}^{c}
$$

where $R_{j}$ is the solid phase of a Boolean model of spheres of radius $r_{j}$ at volume fraction $p_{j}, m$ is the index of the radius threshold $r_{m}$. The sphere radius $r_{j}$ and the corresponding volume fractions $p_{j}$ are chosen according to the experimental pore size distribution.

Second, we consider a Boolean model, denoted by $B$, of spheres of radius $r_{S}$ and volume fraction $p_{S}$ in the 3D domain $D$. This Boolean model is used to locate the aggregates of platelets, independent of the exclusions. Each sphere in the Boolean model is associated with a random orientation $\theta_{l}$, distributed uniformly on unit sphere.

Third, we consider a primary platelet $A^{\prime}$ and implement it randomly inside and outside the aggregates. A Poisson point process generates random points $x_{k}$ with volume fraction of platelets $p_{A 1}$, in the aggregates and out of exclusions $B \cap H^{c}$. At each point, a platelet $A_{x_{k}}^{\prime}$ is located. The platelet is then rotated along the orientation associated with the sphere that it locates in, denoted $A_{x_{k}, \theta_{l}}^{\prime}$. If the platelet is located in the intersection of multiple spheres, it takes an arbitrary orientation of these spheres. The set of aligned platelets inside the aggregates is 


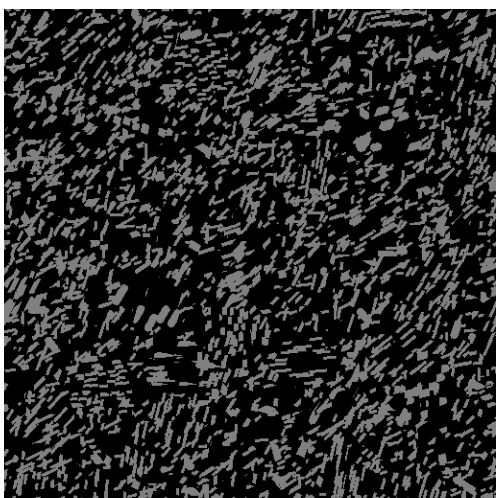

(a) adsorption $r_{p}=0 \mathrm{~nm}$

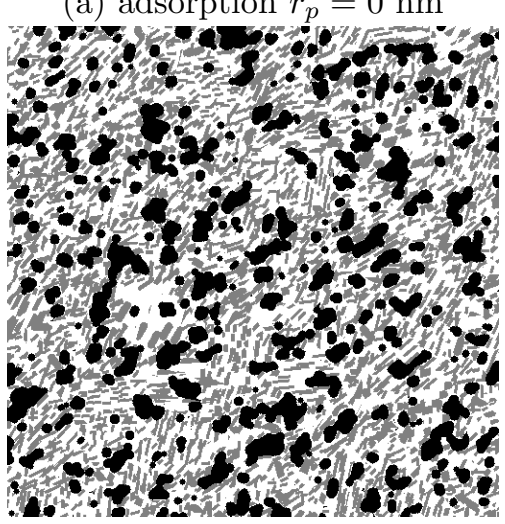

(d) desorption $r_{p}=4 \mathrm{~nm}$

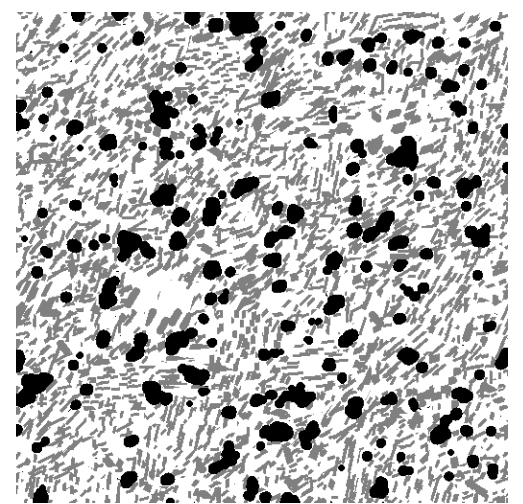

(b) adsorption $r_{p}=5 \mathrm{~nm}$

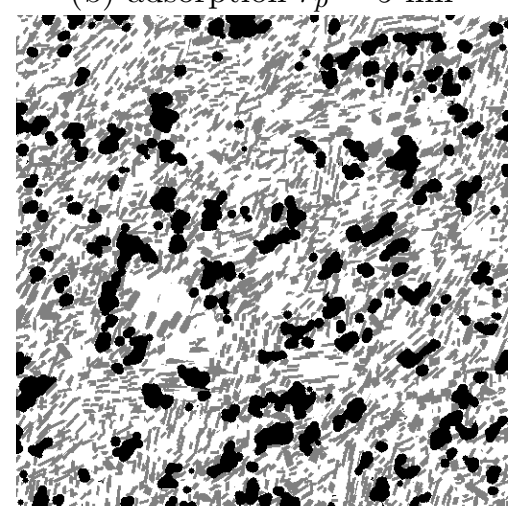

(e) desorption $r_{p}=5 \mathrm{~nm}$

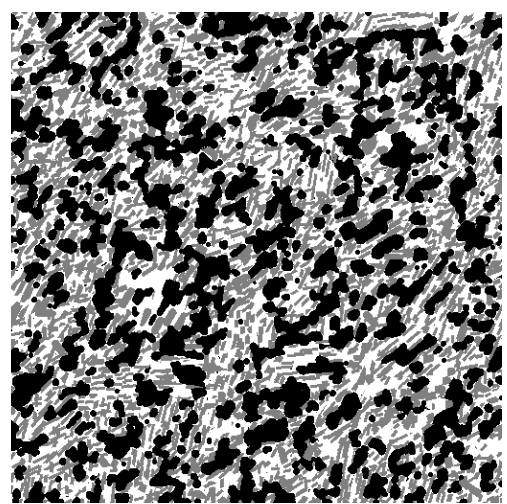

(c) adsorption $r_{p}=6 \mathrm{~nm}$

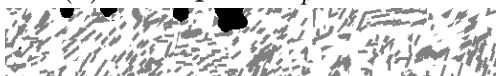
自, Z N (10.4.

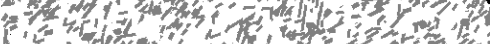

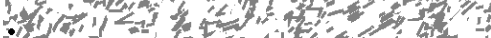
(1) 3h

res

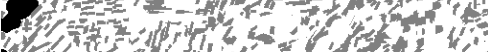
1

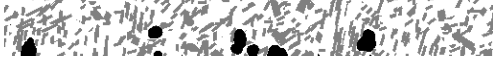

(f) desorption $r_{p}=6 \mathrm{~nm}$

Figure 19: Capillary condensation simulation on the identified two-scale model of platelets of mesoporous alumina (2D sections). Maps (a-c): adsorption; (d-f):desorption.

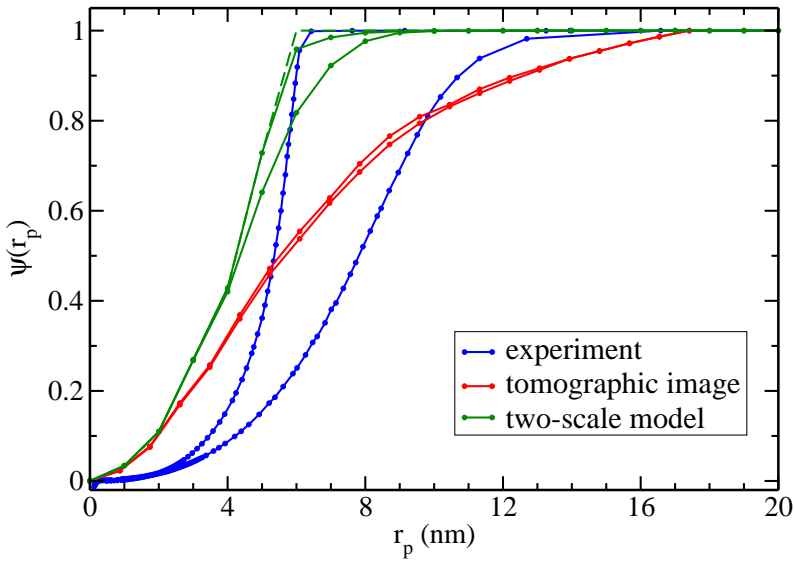

Figure 20: Comparison between the hysteresis of experiment and simulations. 


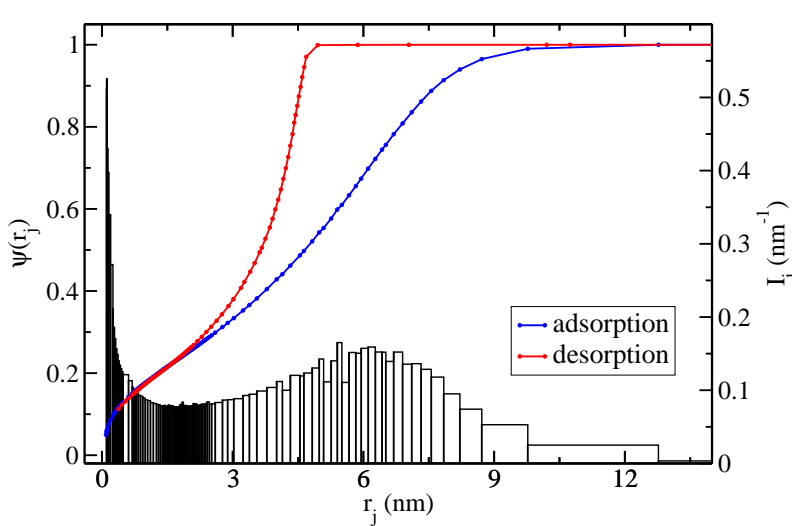

(a)

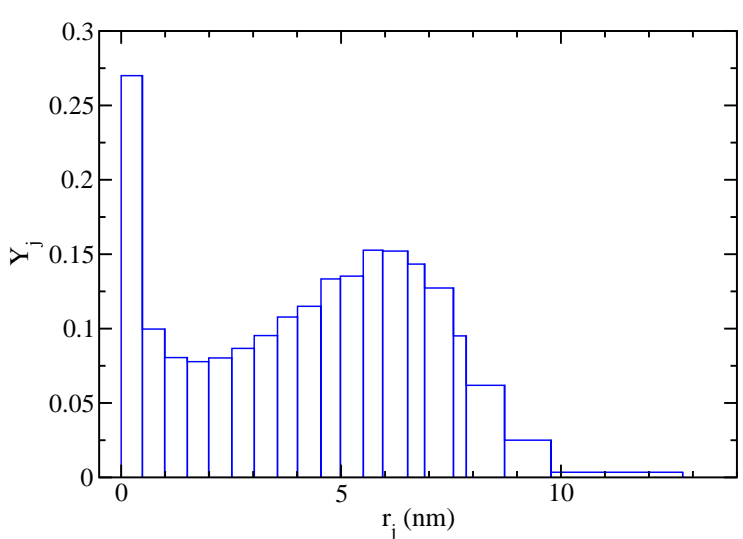

(b)

Figure 21: The pore size distribution deduced directly from the experimental isotherm (a) and its regularization (b).

accordingly:

$$
A_{1}=\bigcup_{x_{k}} A_{x_{k}, \theta_{l}}^{\prime}
$$

Note that the Poisson point process of $x_{k}$ takes place in the domain $B \cap H^{c}$, but the platelets are implemented in the full domain $D$.

A homogeneous Poisson point process is simulated outside the aggregates and the exclusions in $B^{c} \cap H^{c}$. A platelet $A_{y_{k}, \mu_{k}}^{\prime}$ is implanted at each Poisson point $y_{k}$ with volume fraction $p_{B}$. The orientations $\mu_{k}$ are randomly distributed on unit spheres and are independent. The volume fraction $p_{B}$ is prescribed by the overall porosity, experimentally fixed at $69 \%$ :

$$
p_{A 2}\left(1-p_{S}\right)\left(1-p_{H}\right)+p_{A 1} p_{S}\left(1-p_{H}\right)=1-0.69
$$

The set of randomly oriented platelets is given by:

$$
A_{2}=\bigcup_{y_{k}} A_{y_{k}, \mu_{k}}^{\prime}
$$

The three-scale model of platelets, with solid phase denoted by $A$, is obtained by:

$$
A=A_{1} \cup A_{2}
$$

The parameters of the three-scale model $A$ include: radius threshold $r_{m}$, reference pore size distribution $\left(r_{j}, p_{j}\right)$, volume fraction $p_{S}$ and radius $r_{S}$ of spherical aggregates, volume fraction of aligned platelets $p_{A 1}$ and shape of platelets $A^{\prime}$. In this work, we take the octagonal prism as the primary platelet, described by the lengths of four sides (Fig. 16): $D_{1}, D_{2}, D_{3}$ and $D_{4}$.

\subsubsection{Nitrogen porosimetry simulation}

A first microstructure of the three-scale model is generated with the same parameters as the optimized two-scale model in [24]. The radius threshold $r_{m}$ is initially fixed at $0 \mathrm{~nm}$. The reference pore size distribution is deduced from the experimental data by regularization and shown in Fig. 21. The resolution of the microstructure is chosen to be $0.354 \mathrm{~nm}$ per voxel, because it is the size of monolayer nitrogen and it guaranties the necessary precision for the simulation of multilayer adsorption.

The multilayer adsorption at low pressure is simulated first on the microstructure, with $2 \mathrm{D}$ sections shown in Fig. 22a. The number of nitrogen layers on solid interface is fixed at 1 for the initial test. As observed in Fig. 22a, the monolayer nitrogen is "pasted" on the solid walls. Few pores are filled by the monolayer nitrogen. The volume fraction of the monolayer in the total porosity is $11.5 \%$.

After the monolayer adsorption has occurred, the capillary condensation is simulated, starting from Kelvin radius $r_{p}=2 \mathrm{~nm}$. At the corresponding pressure, the small space between platelets begins to be filled. The volume fraction of condensed liquid at this stage is $23 \%$, which is in agreement with the experimental data. Indeed, the volume 


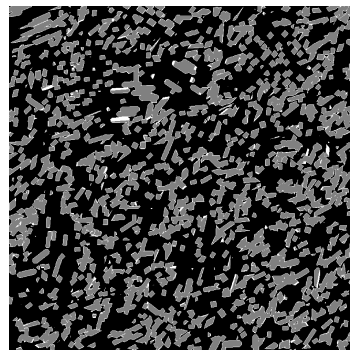

(a)

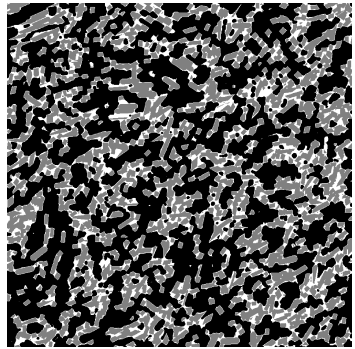

(b)

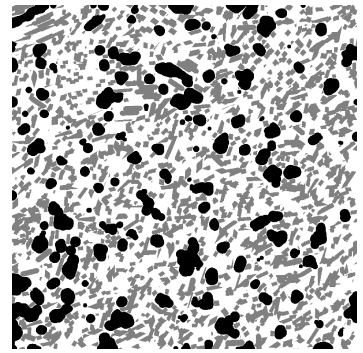

(c)

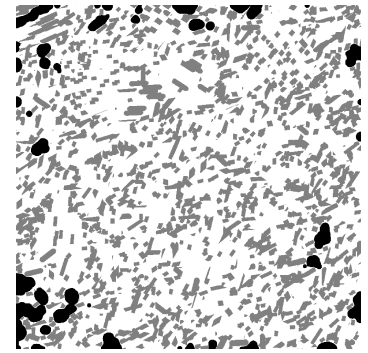

(d)

Figure 22: 2D sections of equilibrium states during porosimetry simulation on the three-scale model of platelets. The parameters of the microstructure are shown in Table 1A. (a) Multilayer adsorption of thickness $r_{t}=0.354 \mathrm{~nm}$. (b) Capillary condensation simulation at $r_{p}=2 \mathrm{~nm}$. (c) Capillary condensation simulation at $r_{p}=4.8 \mathrm{~nm}$. (d) Capillary evaporation simulation at $r_{p}=4.8 \mathrm{~nm}$.

\begin{tabular}{c|cccc|ccc|cc} 
& \multicolumn{4}{|c|}{ Platelets $(\mathrm{nm})$} & \multicolumn{3}{|c|}{ Aggregates } & \multicolumn{3}{c}{ Exclusions } \\
Microstructure & $D_{1}$ & $D_{2}$ & $D_{3}$ & $D_{4}$ & $p_{A 1}$ & $p_{S}$ & $r_{S}$ & $r_{m}(\mathrm{~nm})$ & $\left(r_{j}, p_{j}\right)$ \\
\hline \hline (A) & 14.3 & 2.4 & 2.0 & 3.3 & 0.3 & 0.2 & 30 & 0 & Fig. 21 \\
$(\mathrm{B})$ & 14.3 & 2.4 & 2.0 & 3.3 & 0.3 & 0.2 & 30 & 6.4 & Fig. 21
\end{tabular}

Table 1: Parameter identification of the three-scale model of platelets with only desorption branch. The corresponding simulated isotherms are shown in Fig. 23.

fraction of condensed liquid at low pressure is mainly determined by the specific surface area and the thickness of multilayer nitrogen. The former is dominated by the platelet size. This agreement validates the assumption of only one layer of nitrogen molecule at relative pressure $p / p_{0}=0.6$. It validates also the platelet size identification in our previous work [24].

The simulated isotherms are shown in Fig. 23a. The first point from the origin of the simulated isotherm is the volume fraction of the monolayer at $r_{p}=0.354 \mathrm{~nm}$ (note that here it takes approximately the value of $r_{t}$ ). The second point is the volume fraction of condensed liquid by monolayer adsorption and capillary condensation at $r_{p}=2 \mathrm{~nm}$. The first two points are connected with dotted line instead of solid line, because at such low pressure $\left(r_{p}<2 \mathrm{~nm}\right)$ the Kelvin equation is not valid, and it can only be an approximation.

\subsubsection{Parameter Identification with desorption branch}

A comparison between Fig. 20 and Fig. 23a, shows that the scale of exclusion allows to enlarge the pore size, but this is not sufficient to recover the experimental isotherms. The desorption branch is rather close to the experimental isotherm, but the adsorption branch is still far. Knowing that the Kelvin radius for adsorption is underestimated with the spherical structuring element and that the desorption branch is more reliable (see Section 4), we use only the desorption branch to identify the model parameters. We adjust the value of the radius threshold $r_{m}$ to enlarge more the pore size in the model, and find that $r_{m}=6.4 \mathrm{~nm}$ makes the desorption isotherm fitted to the experimental data with the same desorption threshold. The simulated isotherms is shown in Fig. 23(b). The TEM image simulation method proposed in [23] and refined in [24] is used to validate the three-scale model of platelets, independently from the porosimetry constrained modelling. The correlation function of the simulated TEM image is estimated and compared with the two-scale model and the experimental values in Fig. 24.

The three correlation functions match in general, including the slope at the origin, the intermediate range and the long range. In order to reproduce the correct porosimetry data, exclusions are used to slightly enlarge the pores and the desorption threshold, but as we observe in Fig. 24, it has a side effect that the correlation function of the random structure is also slightly increased. The TEM image simulations bring complementary information on the material microstructure. The three scale model combined to the desorption simulations gives a desorption curve closer to the experimental one (Fig. 23b) and a satisfactory correlation function (Fig. 24b).

\section{Discussion: limitation of the method}

In the capillary adsorption simulation, spherical structuring elements are used to probe the local curvature. Where it cannot enter in, the local curvature is supposed to be smaller than the sphere radius. It fills these areas, 


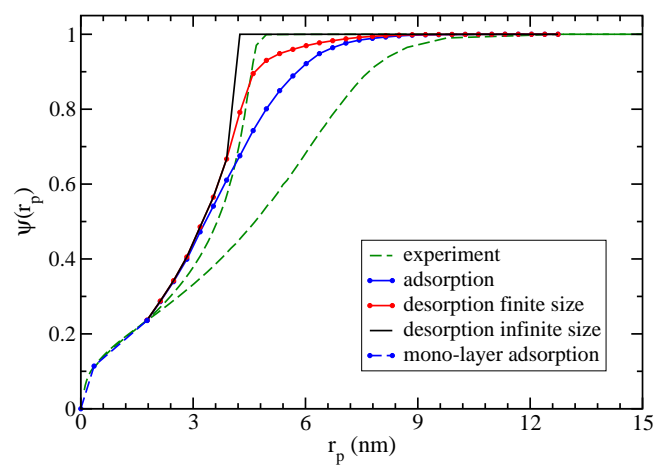

(a)

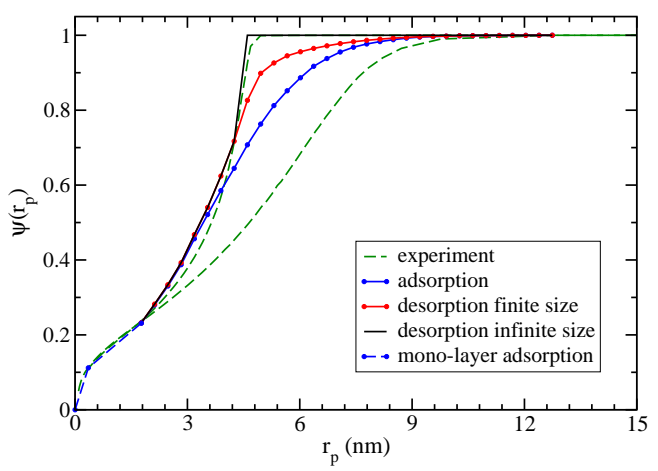

(b)

Figure 23: Simulated nitrogen porosimetry isotherms from the three-scale models of mesoporous alumina with corresponding parameters in Table 1.

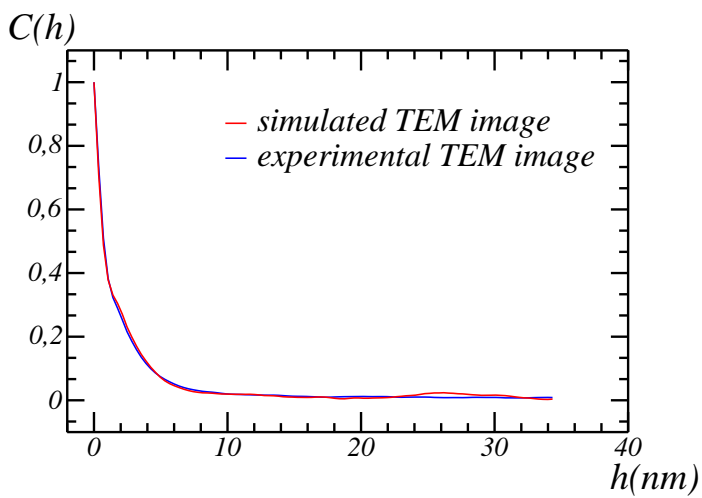

(a)

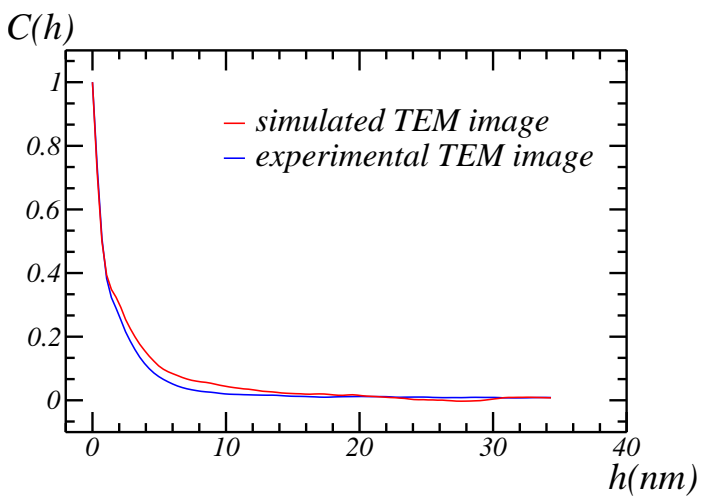

(b)

Figure 24: Experimental and simulated correlation functions of (a) the two-scale model and (b) the three-scale model of platelets for mesoporous alumina. The parameters of the microstructures are shown in Table 1. 


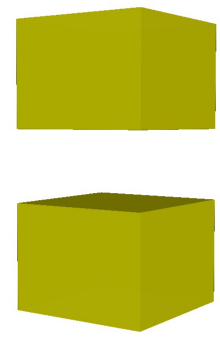

(a)

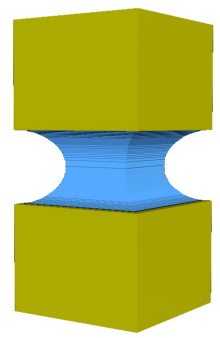

(b)

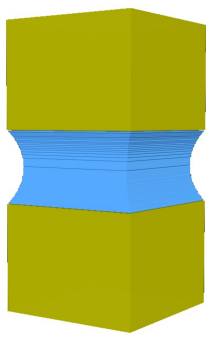

(c)

Figure 25: Condensation $(\mathrm{A} \rightarrow \mathrm{B} \rightarrow \mathrm{C})$ or evaporation $(\mathrm{C} \rightarrow \mathrm{B} \rightarrow \mathrm{A})$ of liquid between two flat surface.

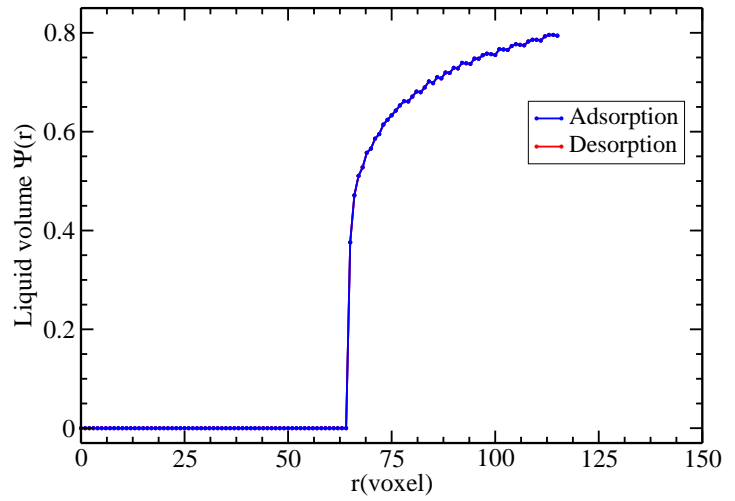

Figure 26: Capillary condensation and evaporation hysteresis curve simulated between two flat surfaces.

and produces hemispherical meniscus on the vapour-liquid interface. This probing process describes the reality during the desorption process, because the entire pore has been filled, and the evaporation depends only on the curvature of the vapour-liquid interface. The vapour-liquid interface tend to be spherical for a minimum energy state. However this is not exactly the case for adsorption, because the condensation depends more on the vapoursolid interface. The vapour-liquid interface tends to be spherical for a minimum energy state. As shown in [3] using a thermodynamic model for an open cylinder [3, 16] making use DFT calculation [16], the liquid-gas meniscus is metastable at adsorption and the condensation pressure does not follow a Kelvin equation. Two examples of this bias are illustrated hereafter: slit pore and the Cohan's model.

\subsection{Slit pore}

We consider a slit pore between two flat surfaces (Fig. 25a). With our method, when nitrogen vapour pressure is low, or more precisely, when the Kelvin radius is less than half of the pore width, no condensation occurs at this stage. When the vapour pressure increases, the equivalent curvature radius becomes larger than half of the pore width, condensation occurs and the meniscus is formed (Fig. 25b). Since the pore is open, without ink-bottle effect, we cannot simulate the hysteresis loop in the isotherm, as shown in Fig. 26.

According to the Kelvin equation, the narrow slit pore between two convex or flat surfaces should not be filled by liquid during adsorption, because the local vapour-solid interface curvature is zero or negative, which already satisfies the Kelvin equation. The meniscus formed with our method also satisfies the Kelvin equation, but it is more realistic for desorption, because during desorption, we consider only the vapour-liquid interface, which remains well spherical. These are two equilibrium states, formed respectively during adsorption and desorption, and create hysteresis without ink-bottle effect. Therefore, the first limitation of the method is that it cannot well find all the equilibrium states in capillary condensation and evaporation.

\subsection{Cohan's model}

Cohan's model [7] is an another example to demonstrate the difference. In a cylindrical pore with one end closed, the condensation occurs first in the corners where the local curvature is low. With our method, hemispherical meniscus is formed, as predicted by the Kelvin equation. The meniscus propagates from the initial meniscus formed in the corners. The entire pore is filled when the Kelvin radius reaches the radius of the cylinder base (denoted 
by $r$ ). In this case, it is right to use a spherical structuring element to probe the local curvature, because the condensation occurs on the hemispherical vapour-liquid interface.

However, in a cylindrical pore with two ends open, the condensation would not occur at the beginning at low pressure. The condensation occurs on the vapour-solid interface. The local mean curvature on the solid interface inside the pore is $1 / 2 r$. The entire pore is filled suddenly when the Kelvin radius reach $2 r$. Cohan predicts hysteresis for the cylindrical pore with two ends open - condensation at $r_{p}=2 r$ and evaporation at $r_{p}=r$. If we use a spherical structuring element to probe the local curvature, the result is $r$, which leads to pore filling for an underestimated pressure.

Cohan's model rightly predicts that hysteresis can be produced even without the ink-bottle effect, in the regime of the Kelvin equation. This effect, the difference in local curvature probing, was not taken into account in our method. It explains the different adsorption pressure observed on the isotherms of ink-bottle model between our method and the method of Virtual Capillary Condensation [19] in Section 2. In a slit pore (Fig. 25), this effect is even more important. The radius of curvature of the flat vapour-solid interface is infinite, and the meniscus curvature during desorption is $d$ (where $d$ is the pore width). The condensation occurs for a saturated pressure, and the evaporation occurs at $r_{p}=d$. Being empty and being filled are two equilibrium states that both satisfy the Kelvin equation. However, the adsorption and the desorption have different preferences between the two states, which brings hysteresis.

The second limitation of the method is therefore the underestimation of the pressures obtained with spherical structuring element during condensation. In order to get closer to reality, the adsorption isotherm should be shifted to the right, by multiplying it by a coefficient 2 if it is a cylindrical pore, infinite if it is a slit pore, or other values depending on the pore morphology.

\section{Conclusion}

In this work, a procedure has been proposed to simulate the capillary condensation and evaporation in porous media. The method is entirely geometrical and relies on morphological operators. The latter is validated on inkbottle pore and on Boolean models. The results are in agreement with the literature. Boundary effects introduce strong finite-size effects on random media. These effects have been corrected by taking into account the percolation threshold of the vapour phase.

The method is applied on the microstructure modelling of mesoporous alumina. Nitrogen isotherms are simulated on existing microstructures using tomographic reconstruction and a two-scale model of platelets. Then, a three-scale model was proposed with Boolean models of spherical exclusions. The parameters of the three-scale model were identified to reproduce the desorption isotherm, and validated independently with TEM visualization. A comparison was made between the behaviours of the two-scale model and the three-scale model in terms of porosimetry and TEM visualization, which shows a consistency between the two independent experiments.

The main advantages of the method include its fast implementation with morphological operations, its efficiency in both computing time and computing space, and its robustness in all geometries. The main limitation of the method is the underestimation of the adsorption pressure, due to the use of spherical elements for the simulation of the condensation step. Use of locally anisotropic adaptive structuring element could improve this part of the curve, at the expense of more complex simulations.

\section{Appendix A. Comparison of the capillary behaviours of some random models of porous media}

\section{Appendix A.1. Boolean models}

In this subsection, we examine how the obstacle shapes affect the capillary condensation isotherms of Boolean models. Instead of spheres, cylinders with different ratio base/height are considered. These models are compared for the same volume fraction of solid phase $p$, the same specific surface area $S_{V}$, the same microstructure volume of $512^{3}$ voxel and the same resolution. The following obstacle shapes are considered: (i) sphere with diameter 20 voxel, as in the previous part of the paper; (ii) oblate cylinder of diameter 40 voxels and height 10 voxels; (iii) intermediate cylinder of diameter 20 voxels and height 20 voxels; (iv) elongated cylinder of radius 15 voxels and height 60 voxels.

The isotherms obtained from the condensation/vapourization simulation are rather close for different obstacle shapes (Fig. A.27). The deduced pore size distribution of the three models is in the same order as the size of obstacles. It is explained by the fact that when the obstacles are uniformly dispersed in the 3D space, the pore size in a Boolean model is mainly determined by the number and size of obstacles in the space, and is not too sensitive 


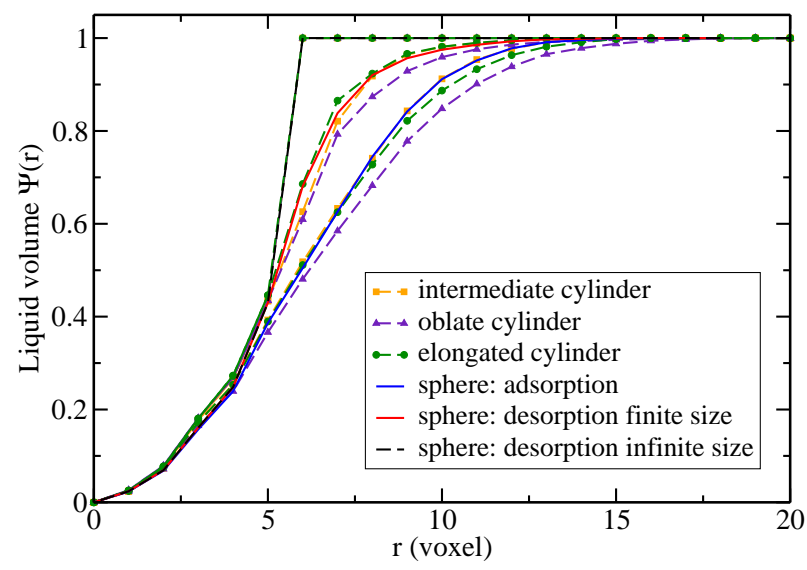

Figure A.27: Capillary condensation/evaporation isotherms in Boolean models of different obstacle shapes.

to the shape of obstacles. When the volume fraction of the obstacles is fixed, the number of obstacles is strongly influenced by the specific surface area, that's to say, the higher the specific surface area is, the smaller the obstacles are, the more obstacles we need to satisfy the volume fraction, and the smaller the pores' sizes are. For a Boolean model, if the volume fraction and the specific surface area are fixed, the pore size distribution is very little affected when the solid phase is obtained by primary grains. It suggests to use other versions of the Boolean model, like pores generated by the primary grains, and by introduction of some multi-scale models that will generate porous media with various size distributions and connectivities.

\section{Appendix A.2. Two-scale Boolean model with spherical pores}

We are interested in the capillary behaviours of multi-scale random models, from which we try to explore new ways to manipulate the isotherms by microstructure modelling. We consider at first a Boolean model of spheres made of two sizes of spheres to generate the pores. It is equivalent to the union of two Boolean models of spheres: a Boolean model of spherical pores with a 36 voxels radius, and pore volume fraction $p_{X}=25 \%$; and a Boolean model of spherical pores with a 12 voxels radius, and pore volume fraction $p_{Y}=25 \%$. These values are chosen because they are lower than the percolation threshold of the Boolean model of equisized spheres of about $29 \%$. The pores percolate but the small spherical pores will block the large pores from releasing during the desorption. The model is a probabilistic version of the combination of regular sphere-cylinder pores in Fig. 6. Its behaviour in capillary condensation simulation is shown in Fig. A.28. The rectangle hysteresis is reproduced in Fig. 6. The small spherical pores play the role of valves, and the critical pore size of the microstructure is the radius of the small pores.

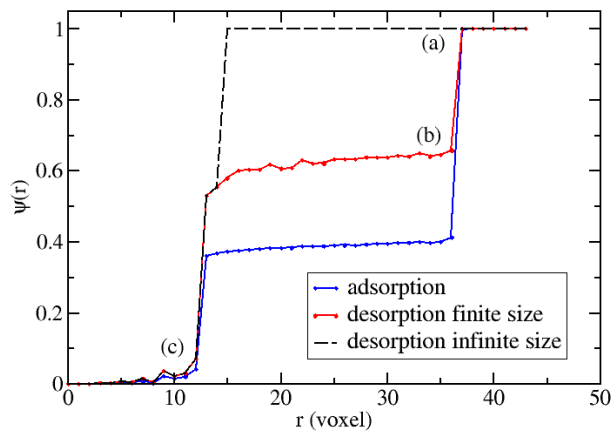

Figure A.28: Capillary condensation and evaporation isotherm of the two-scale model with spherical pores.

From points A to B in Fig. A.29, the large pores connected to the exterior are released, but the entire vapour phase does not percolate. The percolation occurs when the curvature radius reaches $r_{p}=14$ voxel, before the release of most of the small pores. It is explained by the fact that the small pores enlarge the intersection zones between the large pores and improve the connectivity. 


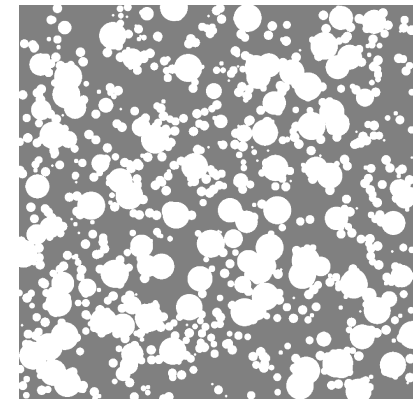

(a)

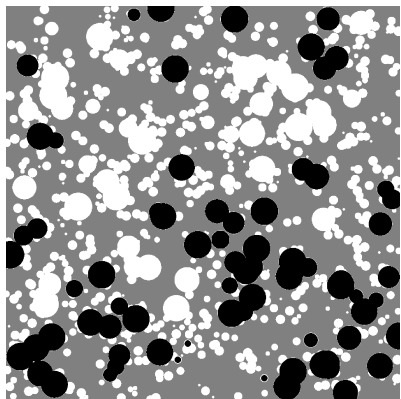

(b)

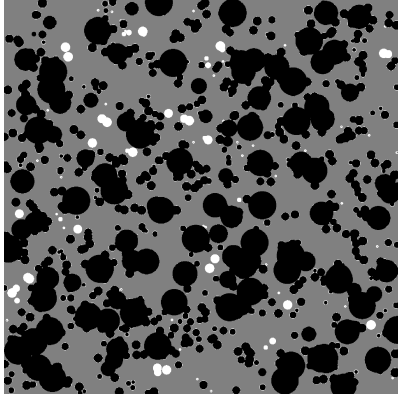

(c)

Figure A.29: Desorption process of the two-scale model with spherical pores. Maps (a), (b) and (c) correspond to $r_{p}=37,36$ and 12 voxels respectively (see Fig. A.28).

Appendix A.3. Two-size hardcore spherical pore model and spherical/cylindrical pores mixture

We now try to eliminate the direct connection between the large pores, so as to highlight the connection functionality of the small pores. In the previous model, we add a repulsion distance ( 6 voxels) between the large spherical pores (with radius 36 voxels). As before, the volume fraction of each size is $25 \%$, and the union of the two models generates a two-scale model of porous medium.

The capillary condensation isotherms simulated on the two-scale model is shown in Fig. A.30. From (a) to (b) in the desorption process, only the pores directly connected to the boundaries are released. The interior of the microstructure remains filled by liquid, until the Kelvin radius reaches the radius of the small spherical pores, and then the whole porosity in the microstructure percolates. A comparison of Figs. A.31b and A.29b shows that the released pores all lie at the boundaries, hence the repulsion distance reduces the connectivity between the large pores. The isotherms in Fig. A.30 is also smoother with less perturbation than that in Fig. A.28.

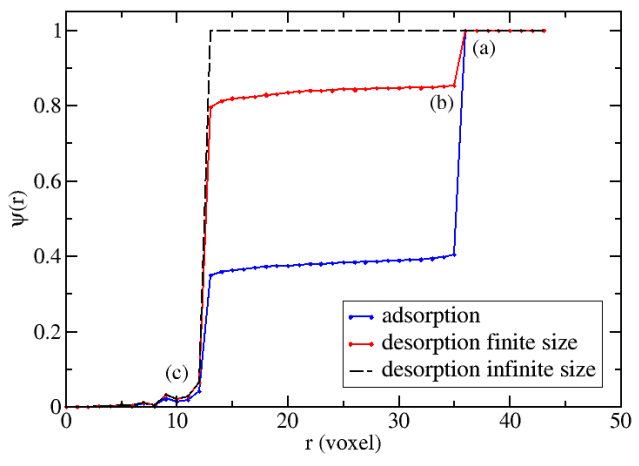

Figure A.30: Capillary condensation and evaporation isotherm of the two-scale model with hardcore spherical pores.

In the above, the threshold for desorption is controlled by the radius of the small pores. Elongated cylinders could also be used to play the role of valves, replacing the spherical pores. We consider again a model made of a union of two models for the pores: first, a Boolean model of spheres of radius 36 voxel, with a volume fraction $p_{X}=25 \%$; second, a model of Poisson fibers with a circular section (radius 12 voxel), with a volume fraction $p_{F}=25 \%$.

The porosimetry isotherm simulated on the two-scale model is shown in Fig. A.32, together with the 2D sections illustrated in Fig. A.33. The capillary behaviours of the two-scale models show that pore size distribution can be manipulated using the combination of various basic models of random sets.

\section{Appendix A.4. Two-scale models obtained by intersections of Boolean models}

Consider first a two-scale model with solid phase $D_{1}=X_{1} \cap X_{2}$ defined as the intersection of two Boolean models of spheres of much different scales $X_{1}$ and $X_{2}$. The spheres in model $X_{1}$ have radius 40 voxels and volume fraction 


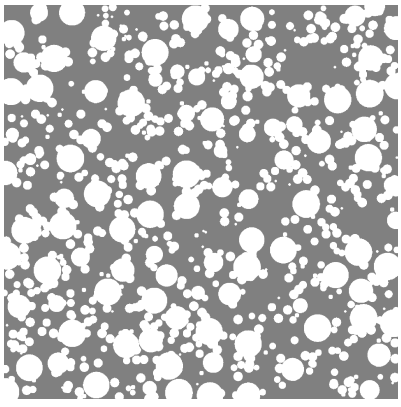

(a)

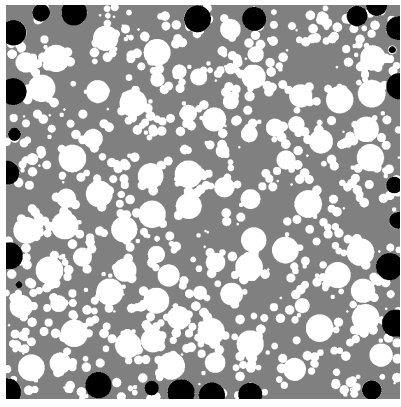

(b)

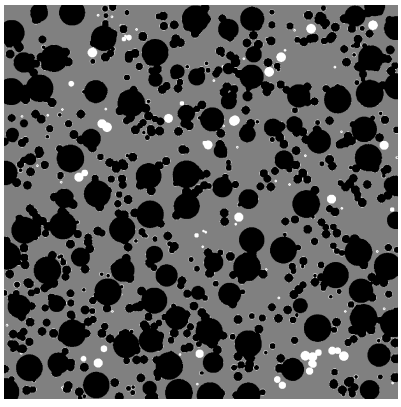

(c)

Figure A.31: Desorption process of the two-scale model with hardcore spherical pores. Maps (a), (b) and (c) correspond to $r_{p}=36,35$ and 12 voxels respectively (see Fig. A.30).

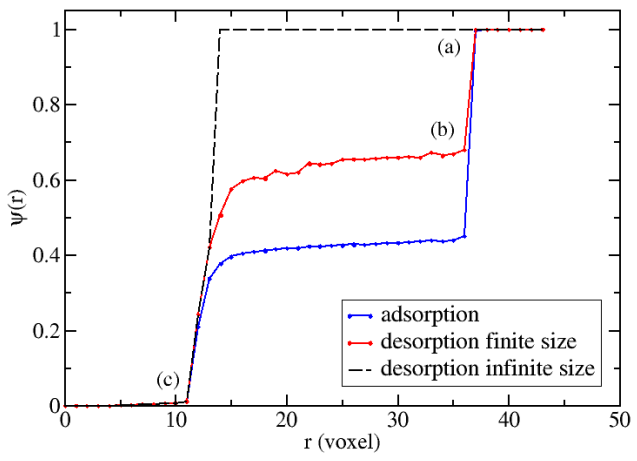

Figure A.32: Capillary condensation/evaporation isotherm of the two-scale model with spherical and cylindrical pores.

$p_{X_{1}}=0.8062$, while the radius of the spheres in model $X_{2}$ is 5 voxels and their volume fraction $p_{X_{2}}=0.8062$. The larger scale plays the role of aggregations, while the small scale plays the role of primary grains. The model is a realistic description for materials like mesoporous alumina [23]. Second, consider a two-scale model with the solid phase $D_{2}=Y_{1}^{c} \cap Y_{2}$ obtained by the intersection of the complementary set of a Boolean model of large spheres and a Boolean model of small spheres. The spheres in model $Y_{1}$ have radius 20 voxels and volume fraction $p_{Y_{1}}=0.1$. In model $Y_{2}$ the spheres radius is 5 voxels and the volume fraction is $p_{Y_{2}}=0.7222$.

For comparison purpose, we also consider a one-scale Boolean model of solid spheres of radius 5 voxel, denoted $D_{3}$, and volume fraction $p_{D_{3}}=0.65$. The pores in this model, $D_{3}^{c}$, are the complementary of $D_{3}$. These three models have the same volume fraction of solid phase $p=0.65$. The parameters of $D_{1}$ are chosen so that the number of large spheres in the cubic volume is the same as the number of small spheres in a large sphere. The volume fraction of large spheres in $D_{2}$ is chosen so that the large spheres do not percolate.

The simulated condensation/vapourization isotherms are shown in Fig. A.34. At low pressure, there is a quick filling of the small pores, and the three models show quantitatively a similar behaviour. When the pore space between small spheres in the model $D_{1}$ was filled, condensed liquid begins a slow filling of the larger pores along with increasing pressure, as shown in Fig. (A.35). The isotherm well combines the same behaviour of two Boolean models at different scales. 


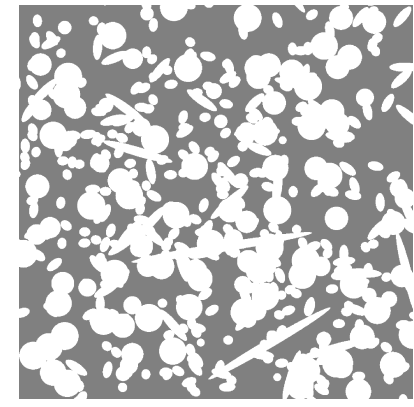

(a)

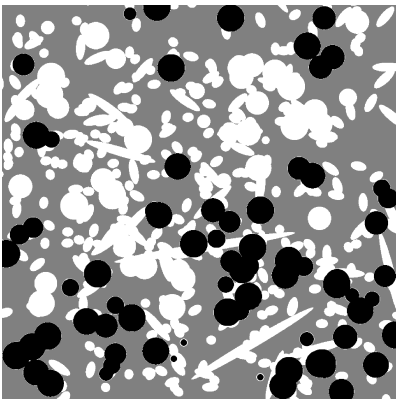

(b)

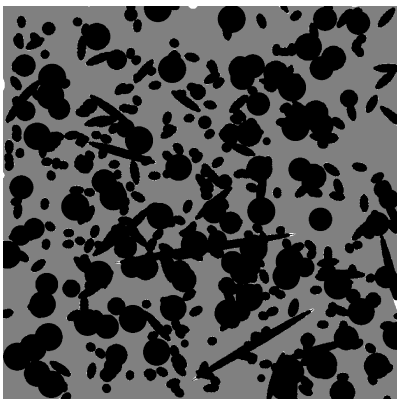

(c)

Figure A.33: Desorption process of the two-scale model with spherical and cylindrical pores. Maps (a), (b) and (c) correspond to $r_{p}=$ 37, 36 and 11 voxels respectively (see Fig. A.32).

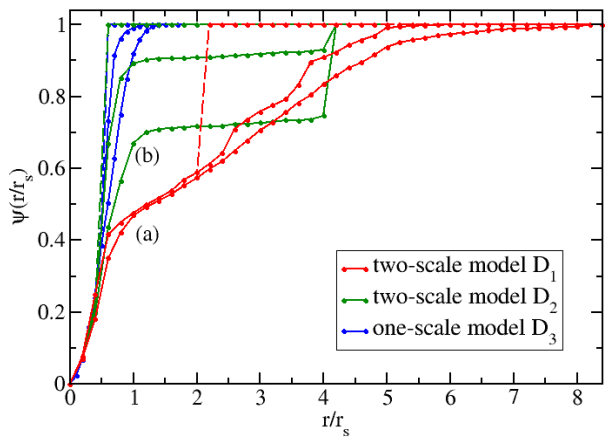

Figure A.34: Capillary condensation isotherm simulated on one-scale and two-scale models.

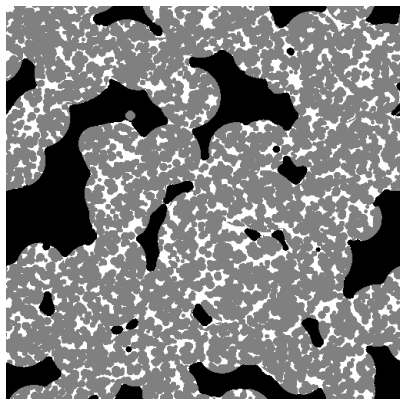

(a)

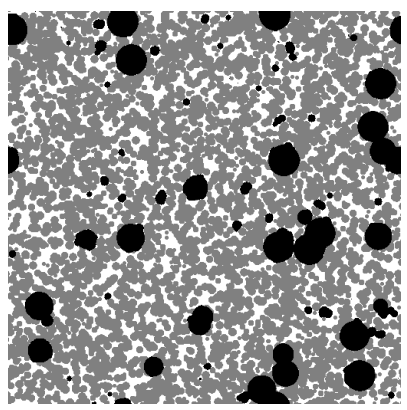

(b)

Figure A.35: 2D section of capillary condensation simulation during the adsorption at $r_{p}=7$ voxel. (a) Two-scale model $D_{1}$. Two-scale model $D_{2}$.

In model $D_{2}$, the scale effect is conspicuous. After the quick filling of the small pores, the large pores remain empty, until the Kelvin radius reaches the radius of the large spherical pores. At the beginning of the desorption process, the large spherical pores are partially released (those connected to the exterior vapour reserve). The pores between small spheres play the role of valves to control the release of the large spherical pores. It is worth mentioning that the percolation threshold of $D_{1}$ is much higher than for $D_{2}$, since the complementary of a Boolean model of spheres has a much lower percolation threshold. 


\section{References}

[1] E. P. Barrett, L. G. Joyner, and P. P. Halenda. The determination of pore volume and area distributions in porous substances. I. computations from nitrogen isotherms. Journal of the American Chemical society, 73(1): 373-380, 1951.

[2] D. P. Bentz and N. S. Martys. Hydraulic radius and transport in reconstructed model three-dimensional porous media. Transport in Porous Media, 17(3):221-238, 1994.

[3] J. C. P Broekhoff and J. H. De Boer. Studies on pore systems in catalysts: IX. calculation of pore distributions from the adsorption branch of nitrogen sorption isotherms in the case of open cylindrical pores A. fundamental equations. Journal of Catalysis, 9(1):8-14, 1967.

[4] S. Brunauer, P. H. Emmett, and E. Teller. Adsorption of gases in multimolecular layers. Journal of the American Chemical Society, 60(2):309-319, 1938.

[5] D. Chiche, M. Digne, R. Revel, C. Chanéac, and J.-P. Jolivet. Accurate determination of oxide nanoparticle size and shape based on X-ray powder pattern simulation: application to boehmite AlOOH. The Journal of Physical Chemistry C, 112(23):8524-8533, 2008.

[6] R. Cimino, K. A. Cychosz, M. Thommes, and A. V. Neimark. Experimental and theoretical studies of scanning adsorption-desorption isotherms. Colloids and Surfaces A: Physicochemical and Engineering Aspects, 437:7689, 2013.

[7] L. H. Cohan. Sorption hysteresis and the vapor pressure of concave surfaces. Journal of the American Chemical Society, 60(2):433-435, 1938.

[8] E. J. Garboczi and D. P. Bentz. Digitised simulation of mercury intrusion porosimetry. Ceramic Transactions, 16:365-380, 1990.

[9] E. Kierlik, P. A. Monson, M. L. Rosinberg, L. Sarkisov, and G. Tarjus. Capillary condensation in disordered porous materials: hysteresis versus equilibrium behavior. Physical Review Letters, 87(5):055701, 2001.

[10] B. Libby and P. A. Monson. Adsorption/desorption hysteresis in inkbottle pores: a density functional theory and Monte Carlo simulation study. Langmuir, 20(10):4289-4294, 2004.

[11] B. C. Lippens, B. G. Linsen, and J. H. De Boer. Studies on pore systems in catalysts i. the adsorption of nitrogen; apparatus and calculation. Journal of Catalysis, 3(1):32-37, 1964.

[12] H. Liu and N. A. Seaton. Determination of the connectivity of porous solids from nitrogen sorption measurements-III. solids containing large mesopores. Chemical engineering science, 49(11):1869-1878, 1994.

[13] G. Matheron. Eléments pour une théorie des milieux poreux. 1967.

[14] C. R. Maurer, R. Qi, and V. Raghavan. A linear time algorithm for computing exact euclidean distance transforms of binary images in arbitrary dimensions. IEEE Transactions on Pattern Analysis and Machine Intelligence, 25(2):265-270, 2003.

[15] B. Münch and L. Holzer. Contradicting geometrical concepts in pore size analysis attained with electron microscopy and mercury intrusion. Journal of the American Ceramic Society, 91(12):4059-4067, 2008.

[16] A. V. Neimark, P. I. Ravikovitch, and A. Vishnyakov. Adsorption hysteresis in nanopores. Physical Review E, 62(2):R1493, 2000.

[17] J. Serra. Image analysis and mathematical morphology, volume 1. Academic Press, 1982.

[18] K. S. W. Sing. Reporting physisorption data for gas/solid systems with special reference to the determination of surface area and porosity (recommendations 1984). Pure and Applied Chemistry, 57(4):603-619, 1985.

[19] F. Štěpánek, M. Šoóš, and P. Rajniak. Characterisation of porous media by the virtual capillary condensation method. Colloids and Surfaces A: Physicochemical and Engineering Aspects, 300(1):11-20, 2007. 
[20] T. Takei, M. Chikazawa, and T. Kanazawa. Validity of the Kelvin equation in estimation of small pore size by nitrogen adsorption. Colloid \& Polymer Science, 275(12):1156-1161, 1997.

[21] J. F. Thovert, J. Salles, and P. M. Adler. Computerized characterization of the geometry of real porous media: their discretization, analysis and interpretation. Journal of Microscopy, 170(1):65-79, 1993.

[22] V.-D. Tran, M. Moreaud, É. Thiébaut, L. Denis, and J.-M. Becker. Inverse problem approach for the alignment of electron tomographic series. Oil \& Gas Science and Technology-Revue dIFP Energies nouvelles, 69(2):279$291,2014$.

[23] H. Wang, A. Pietrasanta, D. Jeulin, F. Willot, M. Faessel, L. Sorbier, and M. Moreaud. Modelling mesoporous alumina microstructure with 3D random models of platelets. Journal of Microscopy, 260(3):287-301, 2015.

[24] H. Wang, F. Willot, M. Moreaud, M. Rivallan, L. Sorbier, and D. Jeulin. Numerical simulation of hindered diffusion in $\gamma$-alumina catalyst supports. Oil $\&$ Gas Science and Technology-Revue dIFP Energies nouvelles, 72(2):8, 2017. 